\title{
CENTRAL SEPARABLE ALGEBRAS WITH PURELY INSEPARABLE SPLITTING RINGS OF EXPONENT ONE
}

\author{
BY \\ SHUEN YUAN( $\left.{ }^{1}\right)$
}

\begin{abstract}
Classical Galois cohomological results for purely inseparable field extensions of exponent one are generalized here to commutative rings of prime characteristic.
\end{abstract}

Given a commutative ring extension $C$ over $A$ of prime characteristic $p$, there are three variants for the Brauer group $B(C / A)$ of central separable $A$-algebras split by $C$ : the Amitsur cohomology group $H^{2}\left(C / A, G_{m}\right)$, the Chase-Rosenberg group $\mathscr{A}(C / A)$, and Hochschild's group $\mathscr{E}(C, \mathfrak{g})$ of regular restricted Lie algebra extensions of $C$ by the Lie algebra $g$ of all $A$-derivations on $C$. In this paper we show that if $C$ is finitely generated projective as an $A$-module and $C[\mathfrak{g}]=\operatorname{End}_{A}(C)$, then $H^{2}\left(C / A, G_{m}\right) \cong \mathscr{E}(\mathfrak{g}, C) \cong \mathscr{A}(C / A)$. As a corollary we show that $H^{\mathrm{t}}\left(C / A, G_{m}\right)$ is zero for all $i>2$. When $C$ is a field, these are the results of Berkson, Hochschild and Rosenberg and Zelinsky [4], [11], [12]. As in [11] we show that the Lie algebra extensions which arise from central separable algebras are trivial extensions when regarded as ordinary extensions so that the essential structural elements are here precisely those which differentiate the restricted extensions from the ordinary ones. We also show that if $R$ is a commutative $C$-algebra which is finitely generated, projective as a $C$-module, then the Brauer group $B(R / A)$ is mapped onto the Brauer group $B(R / C)$. The last result is also due to Hochschild when $C$ is a field [10].

$\S 1$ contains the background on projective modules which came into the picture. Due to their peculiar behavior all relevant automorphisms turn out to be inner which explains why instead of some exact sequences we get two isomorphism theorems. In $\S 2$ the isomorphism of $\mathscr{E}(\mathrm{g}, C)$ with $\mathscr{A}(C / A)$ is proved. $\S 3$ and $\S 4$ provide the preliminary materials for $\$ 5$. $\$ 3$ contains an exposition on the theory of differentials in rings of prime characteristic. Its application to Amitsur cohomology is given in $\$ 4$. The main results are given in $\$ 5$.

Throughout this paper $C$ over $A$ always denotes a commutative ring extension of prime characteristic $p$ such that $C$ is finitely generated projective as an A-module

Received by the editors October 30, 1969.

AMS 1970 subject classifications. Primary 13A20.

Key words and phrases. Regular restricted Lie algebra extensions, central separable algebras, Brauer groups, Amitsur cohomology, derivations, $p$-basis.

(1) Supported in part by National Science Foundation Grant GP-13282.

Copyright (C) 1971, American Mathematical Society 
and $C[\mathfrak{g}]=\operatorname{End}_{A}(C)$ where $\mathfrak{g}=\mathrm{g}(C / A)$ denotes the Lie algebra of all A-derivations on $C$. By a Lie algebra or a Lie algebra homomorphism we mean a Lie algebra or a Lie algebra homomorphism over $A$. The $p$-map of a restricted Lie algebra is denoted by $x \rightarrow x^{p}$, except for two fleeting instants in Lemma 8 and in the discussion preceding Theorem 4 . Tensor product signs without subscripts will denote tensor products over $A$. If $\Theta$ is a subring of a ring $\Xi$, by the $\Theta$-module $\Xi$ we mean the left $\Theta$-module given by the scalar product $\Theta \times \Xi \rightarrow \Xi,(\theta, \xi) \rightarrow \theta \xi$. If $M$ is a $\Theta$-module, then for any $\theta$ in $\Theta$, we will denote by $\Lambda \theta$ the map $M \rightarrow M$ produced by left multiplication by $\theta$. If $M$ is a right $\Theta$-module, the endomorphism on $M$ produced by right multiplication by $\theta$ will be denoted by $P \theta$.

\section{Projective modules}

LEMMA 1. Let $R$ be any commutative A-algebra such that the kernel of $R \otimes R \rightarrow$ $R, x \otimes y \rightarrow x y$, is a nilpotent ideal in $R \otimes R$. Then $H^{0}(R / A, P)=P(R), H^{i}(R / A, P)$ $=0$ for all $i>0$.

Proof. As usual, we denote by $R^{n}$ the tensor product $R \otimes \cdots \otimes R$ ( $n$ factors). Since the kernel of the map $\mu: R^{n} \rightarrow R, x_{1} \otimes \cdots \otimes x_{n} \rightarrow x_{1} \cdots x_{n}$, is nilpotent, we have an isomorphism $P\left(R^{n}\right) \rightarrow P(R)$ given by $M \rightarrow M \otimes_{R^{n}} R \cong M /($ kernel $\mu) M$ [3, p. 90]. Now let $\varepsilon_{i}: R^{n} \rightarrow R^{n+1}$ be the map $x_{1} \otimes \cdots \otimes x_{n} \rightarrow x_{1} \otimes \cdots \otimes x_{i-1} \otimes$ $1 \otimes x_{i} \otimes \cdots \otimes x_{n}$. From the commutativity of

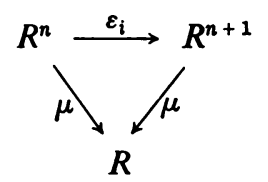

we conclude the commutativity of

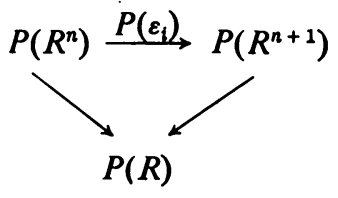

This shows the Amitsur complex

is isomorphic to

$$
\mathscr{C}(R / A, P): 0 \rightarrow P(R) \rightarrow P\left(R^{2}\right) \rightarrow \cdots
$$

$$
0 \longrightarrow P(R) \stackrel{0}{\longrightarrow} P(R) \stackrel{1}{\longrightarrow} P(R) \stackrel{0}{\longrightarrow} P(R) \stackrel{1}{\longrightarrow} \ldots,
$$

whence the lemma.

COROLlaRY. Let $M$ be any rank one projective $C$-module. Then there is an additive group isomorphism $\mathrm{\Upsilon}: C \otimes M \rightarrow C \otimes M$ such that $\Lambda\left(c \otimes c^{\prime}\right) \mathrm{\Upsilon}=$ $\mathrm{r} \Lambda\left(c^{\prime} \otimes c\right), c, c^{\prime} \in C$. 
Proof. The corollary says that every rank one projective $C$-module is a 0 -cocycle in the Amitsur complex $\mathscr{C}(C / A, P)$ which is indeed the case because the kernel of $C \otimes C \rightarrow C, x \otimes y \rightarrow x y$, is nilpotent.

Definition. Let $R$ be a central separable $A$-algebra. By a splitting subalgebra of $R$ we mean a maximal commutative subalgebra $C$ of $R$ such that the $C$-module $R={ }_{C} R$ is projective. It follows from [8, Proposition 2.4] that the map $\omega: C \otimes R^{0} \rightarrow$ End $_{C}(R)$ given by $\omega(x \otimes y) u=x u y, x \in C, y, u \in R$, is an isomorphism.

LeMma 2. Let $R$ be a central separable A-algebra containing $C$ as a subalgebra. If $R_{\mathfrak{q}}$ contains $C_{\mathfrak{q}}$ as a splitting subalgebra for every prime ideal $\mathfrak{q}$ in $A$, then $R$ contains $C$ as a splitting subalgebra.

Proof. Put $C^{\prime}=\{x \in R \mid x c=c x$ for all $c$ in $C\}$. The inclusion map $C \rightarrow C^{\prime}$ is onto because it is onto at every prime ideal $q$ in $A$ [5, p. 111, Theorem 1]. So $C$ is a maximal commutative subalgebra of $R$. Similarly the map $\omega: C \otimes R^{0} \rightarrow \operatorname{End}_{C}(R)$, $c \otimes x \rightarrow(\Lambda c)(P x)$, is an isomorphism because locally it is. Now $C \otimes R^{0} \cong \operatorname{End}_{C}(R)$ implies that (the rank of the $A_{\mathrm{q}}$-module $R_{\mathrm{q}}$ ) $\equiv[R: A]_{\mathrm{q}}=[R: C]_{\mathrm{q}}[C: A]_{\mathrm{q}}$ is equal to $[R: C]_{q}^{2}$. This shows $[R: C]_{\mathbb{Q}}=[R: C]_{Q_{\cap} A}=[C: A]_{Q_{\cap} A}$ is locally constant for $\mathscr{Q}$ in

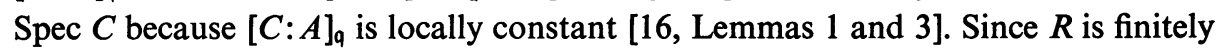
generated as an $A$-module, it is certainly finitely generated as a $C$-module [2, Theorem 2.1]. It follows from [5, p. 138, Theorem 1] that $R$ must be a projective $C$-module.

Let $R$ be any $A$-algebra which contains $C$ as an $A$-subalgebra. We regard $R$ as a $(C \otimes C)$-module via the scalar product $(x \otimes y) v=x v y$.

LEMMA 3. If $R$ is a central separable A-algebra which contains $C$ as a splitting subalgebra, then $R$ is a rank one projective $(C \otimes C)$-module.

Proof. By definition, $R$ is a projective module over $C$. So $R \otimes R^{0}\left({ }_{C} R \otimes R_{C}^{0}\right)$ is a projective $(C \otimes C)$-module. So $R$ is a projective $(C \otimes C)$-module because $R$ is a projective $\left(R \otimes R^{0}\right)$-module.

To show $R$ is a rank one projective $(C \otimes C)$-module, we note that the $A$-module structure on $R$ is obtained from the $(C \otimes C)$-module structure on $R$ by restricting scalars. So for any prime ideal $Q$ in $C \otimes C$, if we write $q=Q \cap A$, we have

$$
\begin{aligned}
{[R: A]_{\mathfrak{q}} } & \equiv\left(\text { rank of } R_{\mathfrak{q}} \text { over } A_{\mathfrak{q}}\right) \\
& =[R: C \otimes C]_{Q}[C \otimes C: A]_{\mathfrak{q}}=[R: C \otimes C]_{Q}[C: A]_{\mathfrak{q}}^{2} .
\end{aligned}
$$

Now $C \otimes R^{0}=\operatorname{End}_{C}(R)$ implies that

$$
[R: C]_{q}^{2}=[R: A]_{q}=[R: C]_{q}[C: A]_{q} \text {. }
$$

So $[R: C]_{\mathfrak{q}}=[C: A]_{\mathfrak{q}}$ and $[R: A]_{\mathfrak{q}}=[C: A]_{q}^{2}$. This shows $[R: C \otimes C]_{Q}=1$ as desired.

LEMMA 4. Let $R$ be a central separable A-algebra which contains $C$ as a splitting subalgebra. Let $J$ denote the kernel of $\mu: C \otimes C \rightarrow C, x \otimes y \rightarrow x y$. Then there is 
an additive group isomorphism $\mathrm{\Upsilon}: C \otimes(R / J R) \rightarrow R$ such that $\mathrm{\Upsilon} \Lambda\left(c \otimes c^{\prime}\right)=$ $\Lambda\left(c^{\prime} \otimes c\right) \Upsilon$ for all $c, c^{\prime}$ in $C$.

Proof. Since $J$ is nilpotent, $\mu$ induces an isomorphism $P(C \otimes C) \rightarrow P(C)$, $M \rightarrow M / J M[3$, p. 90]. Now the map $C \rightarrow C \otimes C, x \rightarrow x \otimes 1$, followed by $\mu$ is the identity on $C$. So any rank one projective $(C \otimes C)$-module $M$ must be isomorphic to $C \otimes(M / J M)=(C \otimes C) \otimes_{C \otimes A}(M / J M)$. The rest of the lemma is trivial.

LEMma 5. Let $R$ be a central separable A-algebra with $C$ as a splitting subalgebra. If the rank one projective class group $P(C / A)$ is trivial, then every automorphism $\sigma$ on $R$ which leaves $C$ pointwise fixed must be inner by some element $z$ in $C$.

Proof. We make $R$ into an $\left(R \otimes R^{0}\right)$-module by defining $(x \otimes y) v=(\sigma x) v y$. Write $M=\{v \in R \mid(\sigma x) v=v x$ for all $x$ in $R\}$. By [2, Theorem 3.1] the map $R \otimes M \rightarrow$ $R, x \otimes v \rightarrow x v$, is an isomorphism. This shows $M R=R M=R$. But $C$ is a $C$-module direct summand of $R$ [5, p. 176, Exercise 4]. So there is a $C$-module homomorphism $\pi: R \rightarrow C$ such that $\pi(c)=c$ for all $c$ in $C$. Now $M$ is an $A$-submodule of $C$ because $v c=c v$ for all $c$ in $C$ and $v$ in $M$. We have $M C=\pi(M R)=\pi(R)=C$. So $M$ is a rank one projective $A$-module split by $C$ and hence must be a free $A$-module because $P(C / A)$ is trivial. Let $z$ be a generator for $M$. It is clear that $\sigma(x)=z x z^{-1}$ for all $x$ in $R$ because $z$ is a unit in $C$.

Now let $r$ be a positive integer. For any ring $X$, we shall denote by $\bar{X}$ the ring $X[t] /\left(t^{r+1}\right)$ of truncated polynomials. Let $R$ be an $A$-algebra and $S$ an $A$-subalgebra of $R$. By a higher $A$-derivation (of rank $r$ ) of $S$ into $R$ we mean an $\bar{A}$-algebra homomorphism $\varphi: \bar{S} \rightarrow \bar{R}, u \rightarrow\left(\varphi_{0} u\right)+\left(\varphi_{1} u\right) t+\cdots+\left(\varphi_{r} u\right) t^{r}, \varphi_{i} u \in R$, such that $\varphi_{0} u=u$ for all $u$ in $S$. A higher $A$-derivation of $R$ into itself is inner if it is an inner automorphism of $\bar{R}$.

LEMMA 6. Let $R$ be a central separable A-algebra containing $C$ as a splitting subalgebra. Then every higher A-derivation $\varphi$ of $C$ into $R$ can be extended to an inner higher derivation of $R$.

Proof. As usual we make $\bar{R}$ into a new $\left(\bar{C} \otimes_{\bar{A}} \bar{R}^{0}\right)$-module ${ }_{\varphi} \bar{R}$ by defining $(u \otimes v) x=(\varphi u) x v$. Write $\Omega=\bar{C} \otimes_{A} \bar{R}^{0}$. By [5, p. 181, Exercise 18] the map $\operatorname{Hom}_{\Omega}\left(\bar{R},{ }_{\varphi} \bar{R}\right) \otimes_{\bar{C}} \bar{R} \rightarrow{ }_{\varphi} \bar{R}, f \otimes u \rightarrow f(u)$, is an isomorphism because $\Omega$ is isomorphic to $\operatorname{End}_{C}(\bar{R})$. Now each element of $\operatorname{Hom}_{\Omega}\left(\bar{R},{ }_{\varphi} \bar{R}\right)$ is determined by its action on $1 \in \bar{R}$ which must go to an element of $M=\{u \in \bar{R} \mid(\varphi x) u=u x$ for all $x \in \bar{C}\}$ because in $\bar{R},(x \otimes 1) \cdot 1=(1 \otimes x) \cdot 1$ for all $x$ in $\bar{C}$. Put $U=\left\{c \in C \mid c+u_{1} t+\right.$ $\cdots+u_{r} t^{r} \in M$ for some $\left.u_{i} \in R\right\}$. We claim that $U=C$ which of course says $\varphi$ can be extended to an inner automorphism of $\bar{R}$. From $M \otimes_{C} \bar{R} \cong_{\varphi} \bar{R}$, it is clear that $U R=R$. But $C$ is a $C$-module direct summand of $R$ [5, p. 176, Exercise 4]. So there is a $C$-module homomorphism $\pi: R \rightarrow C$ such that $\pi c=c$ for all $c$ in $C$. We have $U=U C=\pi(U R)=\pi(R)=C$ as asserted. 
Remark. Given a central separable $A$-algebra $R$ containing $C$ as a maximal commutative subalgebra, if every $\partial \in \mathfrak{g}$ can be extended to an inner derivation of $R$, we will show in Theorem 2 that $C$ is actually a splitting subalgebra of $R$.

2. Regular extensions and central separable algebras. We shall regard $C$ as an abelian restricted Lie algebra with the $p$-map $c \rightarrow c^{P}$. A regular extension of $C$ by $\mathfrak{g}$ is an exact sequence of restricted Lie algebras

$$
L: 0 \longrightarrow C \stackrel{\psi}{\longrightarrow} \mathfrak{l} \stackrel{\varphi}{\longrightarrow} \mathfrak{g} \longrightarrow 0
$$

such that the following conditions are satisfied:

(i) $\psi^{-1}[x, \psi c]=(\varphi x)(c)$ for all $x$ in $\mathfrak{l}$ and $c$ in $C$.

(ii) $\mathfrak{I}$ has a $C$-module structure for which both $\varphi$ and $\psi$ are $C$-linear.

(iii) $\left[c x, c^{\prime} x^{\prime}\right]=c\left(x \cdot c^{\prime}\right) x^{\prime}-c^{\prime}\left(x^{\prime} \cdot c\right) x+c c^{\prime}\left[x, x^{\prime}\right]$ for all $c, c^{\prime}$ in $C$ and $x, x^{\prime}$ in $\mathfrak{l}$ where $x \cdot c=\psi^{-1}[x, \psi c]$.

(iv) $(c x)^{p}=c^{p} x^{p}+D_{c x}^{p-1}(c) x$ for all $c$ in $C$ and $x$ in $\mathfrak{l}$, where $D_{x}$ for any $x$ in $\mathfrak{l}$ denotes the map $c \rightarrow x \cdot c$ on $C$.

Two regular extensions $L, L^{\prime}$ are equivalent if there is a $C$-linear restricted Lie algebra homomorphism $\xi: \mathfrak{l} \rightarrow \mathfrak{l}^{\prime}$ making the diagram

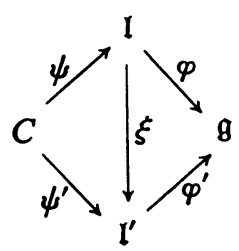

commutative. The set of equivalence classes of regular extensions will be denoted by $\mathscr{E}(\mathfrak{g}, C)$.

We now define an addition on $\mathscr{E}(\mathfrak{g}, C)$. Given two extensions $L$ and $L^{\prime}$, let $D$ denote the subalgebra of the direct sum $\mathfrak{I}+\mathfrak{l}^{\prime}$ which consists of all elements $(u, v)$ in which $\varphi u=\varphi^{\prime} v$. Let $J$ be the ideal of $D$ consisting of the elements $\left(-\psi x, \psi^{\prime} x\right)$ for $x$ in $C$. Since the (pointwise) $p$-map on $D$ maps $J$ into itself, so by Jacobson's formula [7, p. 199, Lemma 2] it induces a well-defined $p$-map on $D / J$. The exact sequence

$$
0 \longrightarrow C \stackrel{\psi^{\prime \prime}}{\longrightarrow} D / J \stackrel{\varphi^{\prime \prime}}{\longrightarrow} \mathfrak{g} \longrightarrow 0, \quad x \stackrel{\psi^{\prime \prime}}{\longrightarrow}(\psi x, 0)+J, \quad(u, v)+J \stackrel{\varphi^{\prime \prime}}{\longrightarrow} \varphi u
$$

is a regular extension of $C$ by $g$ and is defined to be the sum of $L$ and $L^{\prime}$. It is a straightforward matter to verify that $\mathscr{E}(\mathfrak{g}, C)$ form an abelian group with respect to this addition. The 0 element is the trivial extension $0 \rightarrow C \rightarrow C+g \rightarrow g \rightarrow 0$ where $C+\mathfrak{g}$ is the $C$-module direct sum of $C$ and $\mathfrak{g}$ with the commutation $\left[(x, \partial),\left(x^{\prime}, \partial^{\prime}\right)\right]=\left(\partial x^{\prime}-\partial^{\prime} x,\left[\partial, \partial^{\prime}\right]\right)$ and the $p$-map $(x, \partial) \rightarrow\left(x^{p}+\partial^{p-1} x, \partial^{p}\right)$. The negative of a regular extension $L$ is just $0 \rightarrow C \rightarrow \mathfrak{l} \rightarrow \mathfrak{g} \rightarrow 0$.

Now let $R$ be a central separable $A$-algebra containing $C$ as a maximal commutative subalgebra such that every $\partial$ in $\mathfrak{g}$ can be extended to an inner derivation 
on $R$. Let $\mathfrak{l}$ denote the set of all elements $s$ of $R$ for which $D_{s}(C) \subset C$ where $D_{s}$ denotes the derivation of $R$ which is given by $D_{s} x=s x-x s$ for all $x$ in $R$. Let $\varphi(s)$ denote the restriction of $D_{s}$ to $C$. The $C$-module $\mathfrak{l}$ carries a restricted Lie algebra structure with $\left[s_{1}, s_{2}\right]=s_{1} s_{2}-s_{2} s_{1}$ and the $p$-map $s \rightarrow s^{p}$. The $C$-linear restricted Lie algebra homomorphism $s \rightarrow \varphi(s)$ maps $\mathfrak{l}$ onto all of $\mathfrak{g}$ and clearly the kernel of $\varphi$ coincides with $C$. So we have an exact sequence of restricted Lie algebras $L: 0 \rightarrow C \hookrightarrow \mathfrak{l} \stackrel{\oplus}{\rightarrow} \mathfrak{g} \rightarrow 0$. It follows from [11, Lemma 1] that $L$ is a regular extension.

THEOREM 1 (WEAK Form). If there is an A-derivation $\partial$ on $C$ such that $C[\partial]=$ End $_{A}(C)$, then every regular extension $L: 0 \rightarrow C \hookrightarrow \stackrel{\mathfrak{l}}{\rightarrow} \mathfrak{g} \rightarrow 0$ admits $a$ C-linear Lie algebra homomorphism $\rho: \mathfrak{g} \rightarrow \mathfrak{l}$ such that $\varphi \rho$ is the identity map on $\mathfrak{g}$. For any such $\rho$, the map $\theta: \mathfrak{g} \rightarrow A, d \rightarrow \rho(d)^{p}-\rho\left(d^{p}\right)$, is additive and $\theta(u d)=u^{p}(\theta d)$ for all $u \in C$.

Proof. We observe that given any idempotent $e$ in $A, \mathfrak{l}=e \mathfrak{l}+(1-e) \mathfrak{l}$ is a direct sum of restricted Lie algebras. In view of the argument given in [15, Corollary 2.5] we may therefore assume, without loss of generality, that the projective $A$-module $C$ has a rank $p^{n}$. It follows from [15, Theorem 2.4] that $\partial$ satisfies a polynomial $X=\alpha_{0} t+\cdots+\alpha_{i} t^{p^{t}}+\cdots+\alpha_{n-1} t^{p^{n-1}}+t^{p^{n}}, \alpha_{i} \in A$, and any polynomial $f$ in $C[t]$ satisfied by $\partial$ is divisible by $X$. Let $g_{0}$ denote the abelian Lie subalgebra $\sum_{i=0}^{n-1} A \partial^{p^{\imath}}$ of $\mathfrak{g}$. Let $s$ be any element in $\mathfrak{l}$ such that $\varphi(s)=\partial$. It follows from the identity $D_{s^{p}}=D_{s}^{p}$ that $\sum_{i=0}^{\infty} A s^{p^{4}}$ is an abelian Lie subalgebra of $\mathfrak{l}$. Let $\rho: \mathfrak{g}_{0} \rightarrow \mathfrak{l}$ be the $A$ module homomorphism given by $\partial^{p^{t}} \rightarrow s^{p^{t}}$. Since $\partial^{p^{t}}, i=0, \ldots, n-1$, are linearly independent over $C, \rho$ is well defined and is clearly a Lie algebra homomorphism. Moreover, $\varphi \rho$ is the identity map on $g_{0}$. Now the $C$-module monomorphism $C \otimes g_{0} \rightarrow g, u \otimes d \rightarrow u d$, is actually onto because locally it is always onto [15, p. 44, Proof of Lemma 2.2]. We may therefore extend $\rho$ to a $C$-module homomorphism (still denoted by $\rho$ ) from $g$ to $\mathfrak{l}$ by setting $u d \rightarrow u(\rho d), u \in C, d \in g_{0}$. It follows from the first and third regularity conditions assumed on $L$ that $\rho: \mathfrak{g} \rightarrow \mathfrak{l}$ is a Lie algebra homomorphism. That $\rho$ followed by $\varphi$ is the identity map on $g$ is of course clear. We claim that $\theta: d \rightarrow \rho(d)^{p}-\rho\left(d^{p}\right), d \in \mathfrak{g}$, takes its values in $A$. We have for any $d^{\prime} \in \mathfrak{g}$,

$$
-d^{\prime}(\theta d)+\rho\left[d^{p}, d^{\prime}\right]=D_{\theta(d)+\rho\left(d^{p}\right)}\left(\rho d^{\prime}\right)=D_{\rho(d)^{p}}\left(\rho d^{\prime}\right)=D_{\rho(d)}^{p}\left(\rho d^{\prime}\right)=\rho\left(D_{d}^{p}\left(d^{\prime}\right)\right) .
$$

So $d^{\prime}(\theta d)=0$ because $\left[d^{p}, d^{\prime}\right]=D_{d}^{p}\left(d^{\prime}\right)$ by definition of restricted Lie algebra. This shows $\theta(d) \in A$ for all $d$ in $\mathfrak{g}$. Next, given $d$ and $d^{\prime}$ in $\mathfrak{g}$, according to [7, p. 199-200, (22), (28)], $\left(d+d^{\prime}\right)^{p}-d^{p}-d^{\prime p}$ is a certain sum of commutators formed from $d$ and $d^{\prime}$. So $\rho\left(d+d^{\prime}\right)^{p}-\rho(d)^{p}-\rho\left(d^{\prime}\right)^{p}=\rho\left(\left(d+d^{\prime}\right)^{p}\right)-\rho\left(d^{p}\right)-\rho\left(d^{\prime p}\right)$ from which we conclude that $\theta\left(d+d^{\prime}\right)=\theta(d)+\theta\left(d^{\prime}\right)$. Finally for any $u$ in $C$, we have, by Hochschild's formula [11, Lemma 1] and the $C$-linearity of $\rho$,

$$
\begin{aligned}
\theta(u d) & =\rho(u d)^{p}-\rho\left((u d)^{p}\right) \\
& =\left(u^{p}(\rho d)^{p}+\left((u d)^{p-1} u\right)(\rho d)\right)-\rho\left(u^{p} d^{p}+\left((u d)^{p-1} u\right) d\right) \\
& =u^{p}\left((\rho d)^{p}-\rho\left(d^{p}\right)\right)=u^{p}(\theta d) .
\end{aligned}
$$

This completes the proof of the theorem. 
COROllary. If $C$ admits a p-basis over $A$, then every regular extension $L: 0 \rightarrow$ $C \rightarrow \stackrel{I}{\rightarrow} \rightarrow \mathfrak{g} \rightarrow 0$ admits a C-linear Lie algebra homomorphism $\rho: \mathfrak{g} \rightarrow \mathfrak{l}$ such that $\varphi \rho$ is the identity on $\mathrm{g}$.

The corollary is an immediate consequence of the following:

Lemma 7. If $C$ admits a p-basis $u_{i}, 1 \leqq i \leqq r$, over $A$, then the A-derivation on $C$ given by $\partial u_{1}=1, \partial u_{i}=\left(u_{1} \cdots u_{i-1}\right)^{p-1}, i>1$, satisfies the equation $C[\partial]=\operatorname{End}_{A}(C)$.

Proof. Let $S$ be the set of all monomials $u_{1}^{e_{1}} \cdots u_{r}^{e_{r}}, 0 \leqq e_{i}<p$. We impose an order on $S$ as follows: $u_{1}^{l_{1}} \cdots u_{r}^{l_{r}}<u_{1}^{m_{1}} \cdots u_{r}^{m_{r}}$ if and only if for some $k, l_{k}<m_{k}$ and $l_{i}=m_{i}$ for all $i>k$. Let $\omega$ be the one-to-one order-preserving function from $S$ into the set of all positive integers given by $\omega(1)=1, \omega\left(\left(u_{1} \cdots u_{r}\right)^{p-1}\right)=p^{r}$. For any $s$ in $S$, we assert that $\partial^{\omega(s)} s=0, \partial^{\omega(s)-1}(s) \in\{1,2, \ldots, p-1\}$. This is clear if $\omega(s)=1$. If $\omega(s)>1$, then $s$ is of the form $u_{m}^{e_{m}} \prod_{m<i \leqq r} u_{i}^{e_{i}}$ with $e_{m}>0$. We have

$$
\partial s=e_{m}\left(\partial u_{m}\right) u_{m}^{e_{m}-1} \prod_{m<i \leqq r} u_{i}^{e_{i}}+\sum_{j=m+1}^{r} e_{j}\left(\partial u_{j}\right) u_{m}^{e_{m}} u_{j}^{e_{j}-1} \prod_{i \neq j: m<i \leqq r} u_{i}^{e_{i}}
$$

Put $t=\left(\partial u_{m}\right) u_{m}^{e_{m}-1} \prod_{m<i \leqq r} u_{i}^{e_{i}}$. It is clear that $\omega(t)=\omega(s)-1$. It is also clear that monomials occurring in the summation sign are strictly less than $t$. So by an inductive argument, we see that $\partial^{\omega(s)} s=0, \partial^{\omega(s)-1} s \in\{1,2, \ldots, p-1\}$.

Now let $a$ be any ideal in $C$. We claim that if $\sum_{0 \leqq i<p^{r}} c_{i} \partial_{i}=f$ for some $c_{i}$ in $C$ and $f$ in $\mathfrak{a E n d}_{A}(C)$, then $c_{i}$ belongs to $\mathfrak{a}$ for all $i$. Assume $c_{i}$ belongs to $\mathfrak{a}$ for all $i<l$. Let $s \in S$ be the monomial with $\omega(s)=l+1$. It follows from $c_{l}\left(\partial^{l} s\right)=f(s)-$ $\sum_{\mathfrak{i}<l} c_{\mathfrak{i}}\left(\partial^{i} s\right) \in \mathfrak{a}$ that $c_{l}$ belongs to $\mathfrak{a}$ also because $\partial^{l} s$ is invertible. If we take $\mathfrak{a}$ to be the zero ideal in $C$, we see that $C[\partial]=\sum_{0 \leqq i<p^{r}} C \partial^{i}$ is a free $C$-module based on $\partial^{i}$, $0 \leqq i<p^{r}$. Moreover the canonical map $C[\partial] / \mathfrak{a} C[\partial] \rightarrow \operatorname{End}_{A}(C) / \mathfrak{a} \operatorname{End}_{A}(C)$ is a monomorphism for any ideal $\mathfrak{a}$ in $C$. If $\mathfrak{a}$ is a maximal ideal of $C$, then $C[\partial] / \mathfrak{a} C[\partial] \rightarrow$ $\operatorname{End}_{A}(C) / \mathfrak{a} \operatorname{End}_{A}(C)$ is an isomorphism because both sides are vector spaces over $C / \mathfrak{a}$ of the same finite dimension $p^{r}=[C: A]$. In particular $C[\partial]=\operatorname{End}_{A}(C)$ when $C$ is a local ring [5, p. 109, Corollary]. So the inclusion map $C[\partial] \rightarrow \operatorname{End}_{A}(C)$ is always onto because locally it is [5, p. 111, Theorem 1].

REMARK. (a) The restriction in Theorem 1 that there is a single derivation $\partial$ with $C[\partial]=\operatorname{End}_{A}(C)$ will be removed later in $\$ 5$.

(b) In case $C$ is a field, the above corollary is due to Hochschild [11, Theorem 4]. Our proof is somewhat easier because we make no use of the cohomology of restricted Lie algebras.

Let $\mathfrak{I}$ be a restricted Lie algebra with the $p$-map $x \rightarrow x^{[p]}$ and denote by $J$ the ideal of the universal enveloping algebra $R_{\mathfrak{l}}$ of $\mathfrak{l}$ generated by the elements of the form $x^{p}-x^{[p]}$ with $x$ in $\mathfrak{l}$. It follows from the definition of restricted Lie algebras that these elements are in the center of $R_{\mathfrak{l}}$. We define the restricted enveloping algebra of $\mathfrak{l}$ to be $U_{\mathfrak{l}}=R_{\mathfrak{l}} / J$. Let $I(\mathfrak{l})$ denote the ideal in $U_{\mathfrak{l}}$ generated by the canonical image of $\mathfrak{l}$. Then we have an $A$-algebra homomorphism $\varepsilon: U_{\mathfrak{l}} \rightarrow A=U_{\mathfrak{l}} / I(\mathfrak{l})$. So $U_{\mathrm{l}}$ is a supplemented algebra in the terminology of [6]. 
LEMMA 8. Let $\mathfrak{l}$ be a restricted Lie algebra such that $\mathfrak{l}$ is a finitely generated free $A$-module based on $\lambda_{1}, \ldots, \lambda_{r}$. Then the monomials $\lambda_{1}^{e_{1}} \cdots \lambda_{r}^{e_{r}}, 0 \leqq e_{i}<p$, form an A-module basis for $U_{\mathrm{l}}$.

Proof. By [6, p. 271, Theorem 3.1], the monomials $\lambda_{1}^{e_{1}} \cdots \lambda_{r}^{e_{r}}, e_{i} \geqq 0$, form an $A$-module basis for $R_{\mathfrak{l}}$ over $A$. Put $\mu_{i}=\lambda_{i}^{p}-\lambda_{i}^{[p]}$. A simple verification shows that the monomials $\lambda_{1}^{m_{1}} \cdots \lambda_{r}^{m_{r}} \mu_{1}^{n_{1}} \cdots \mu_{r}^{n_{r}}, 0 \leqq m_{i}<p, n_{i} \geqq 0$, form a basis for $R_{\mathrm{l}}$ over $A$. So the monomials $\lambda_{1}^{e_{1}} \cdots \lambda_{r}^{e_{r}}, 0 \leqq e_{i}<p$, form a basis for $U_{\mathfrak{l}}$ over $A$.

LEMMA 9. Let $\mathfrak{l}$ be a restricted Lie algebra such that $\mathfrak{l}$ is finitely generated projective as an A-module. Then $U_{\mathrm{l}}$ is also finitely generated projective as an A-module. Moreover, $\mathfrak{l}$ is a direct summand of $I(\mathfrak{l})$.

Proof. Since $\mathfrak{l}$ is finitely generated projective over $A$, given any maximal ideal $\mathfrak{q}$ in $A$ there is some $f \in A-\mathfrak{q}$ such that $\mathfrak{l}_{f}$ is a free $A_{f}$-module of finite dimension. Now $\left(U_{\mathfrak{l}}\right)_{f}$ may be identified as the restricted enveloping algebra of $\mathfrak{l}_{f}$ over $A_{f}$ so it must be a finite dimensional free $A_{f}$-module. This implies $U_{\mathfrak{l}}$ is finitely generated projective over $A$.

Next the canonical map $\mathfrak{l} \rightarrow U_{\mathfrak{l}}$ is a monomorphism because it is a monomorphism locally. So we may regard $\mathfrak{l}$ as an $A$-submodule of $I(\mathfrak{l})$. In view of [5, p. 114, Corollary 1] to show $\mathfrak{l}$ is a direct summand of $I(\mathfrak{l})$, it suffices to show $I(\mathfrak{l}) / \mathfrak{l}$ is finitely presented as an $A$-module. But $I(\mathfrak{l})$ as a direct summand of $U_{\mathfrak{l}}$ is finitely generated projective over $A$, so there is a finitely generated projective $A$-module $J$ such that the direct sum $I(\mathfrak{l})+J$ is a free $A$-module. It follows from the exactness of the sequence

$$
0 \rightarrow \mathfrak{l}+J \hookrightarrow I(\mathfrak{l})+J \rightarrow I(\mathfrak{l}) / \mathfrak{l} \rightarrow 0, \quad(x, y) \rightarrow x+\mathfrak{l},
$$

that $I(\mathfrak{l}) / \mathfrak{l}$ is finitely presented because $\mathfrak{l}+J$ is finitely generated as an $A$-module.

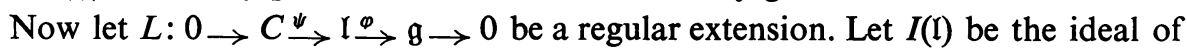
the restricted enveloping algebra $U_{\mathfrak{l}}$ of $\mathfrak{l}$ which is generated by the canonical images $x^{\prime}$ in $U_{\mathfrak{l}}$ of the elements $x$ in $\mathfrak{l}$. Let $J$ be the ideal of $I(\mathfrak{l})$ which is generated by the elements $(\psi c)^{\prime} x^{\prime}-(c x)^{\prime}$ where $c \in C, x \in \mathfrak{l},(\psi c)^{\prime} x^{\prime}$ denotes the product of $(\psi c)^{\prime}, x^{\prime}$ in $U_{\mathfrak{l}}, c x$ denotes the scalar product in $\mathfrak{l}$. Put $V_{L}=I(\mathfrak{l}) / J$. It is clear that $V_{L}$ is essentially determined by the equivalence class of $L$.

THEOREM 2. Let $L: 0 \rightarrow C \hookrightarrow \mathfrak{l} \stackrel{\oplus}{\rightarrow} \mathfrak{g} \rightarrow 0$ be a regular extension. Then the canonical map of $\mathfrak{l}$ into $V_{L}$ is one-one, and its restriction to $C$ is a ring-homomorphism by means of which we identify $C$ with a subring of $V_{L}$. Then $V_{L}$ is a central separable A-algebra containing $C$ as a splitting subalgebra. Furthermore $\mathfrak{l}$ becomes identified by the canonical map with the set $\left\{x \in V_{L} \mid x c-c x \in C, \forall c \in C\right\}$ and the corresponding regular extension coincides with $L$. Conversely, if $R$ is a central separable A-algebra containing $C$ as a maximal commutative subalgebra such that every $\partial$ in $g$ can be extended to an inner derivation of $R$ and if $L$ is the corresponding regular extension, then $V_{L}$ is isomorphic to $R$. 
Proof. Given any prime ideal $\mathfrak{q}$ in $A$ it is easy to verify that $\left(V_{L}\right)_{\mathfrak{q}}$ is isomorphic to $V_{L \mathfrak{q}}$ where $L_{\mathfrak{q}}$ is the regular extension $0 \rightarrow C_{\mathfrak{q}} \hookrightarrow \mathfrak{I}_{\mathfrak{q}} \rightarrow \mathfrak{g}_{\mathfrak{q}} \rightarrow 0$. It follows from Lemma 2 that all the assertions in the theorem are local in nature, it suffices to prove the theorem under the assumption that $A$ is a local ring. So $C$ admits a $p$-basis $u_{i}, 1 \leqq i \leqq r$, over $A$ [16, Lemma 7 and Theorem 10]. Let $\partial_{i}$ be the $A$-derivation on $C$ given by $\partial_{i} u_{j}=\delta_{i j} u_{j}$. By the corollary to Theorem 1 there is an ordinary Lie algebra homomorphism $\rho: \mathfrak{g} \rightarrow \mathfrak{l}$ which is $C$-linear and such that for every $\partial \in \mathfrak{g},(\varphi \rho) \partial=\partial$ and $\rho(\partial)^{p}-\rho\left(\partial^{p}\right) \in A$. Write $s_{i}=\rho\left(\partial_{i}\right)$. Then $s_{i}^{p}=s_{i}+\alpha_{i}$ with $\alpha_{i} \in A$ because $\partial_{i}^{p}=\partial_{i}$. In the polynomial ring $A\left[t_{1}, \ldots, t_{r}\right]$ where the $t_{i}$ are indeterminates over $A$, let $I$ be the ideal generated by the elements $t_{i}^{p}-t_{i}-\alpha_{i}$. Put $Z=A\left[t_{1}, \ldots, t_{r}\right] / I$. Then if $z_{i}$ denotes the cosets of $t_{i}$ modulo $I$, we have $Z=A\left[z_{1}, \ldots, z_{r}\right], z_{i}^{p}=z_{i}+\alpha_{i}$ and the monomials $z_{1}^{e_{1}} \cdots z_{r}^{e_{r}}$ with $0 \leqq e_{i}<p$ constitute a basis for $Z$ over $A$. Now put $W=C \otimes Z$. Let $y_{i}$ be the $A$-module endomorphism $\partial_{i} \otimes 1+1 \otimes \Lambda z_{i}$ of $W$ where $\Lambda z_{i}: Z \rightarrow Z$ is the map produced by left multiplication by $z_{i}$. Let $R=$ $C\left[y_{1}, \ldots, y_{r}\right] \subset \operatorname{End}_{A}(W)$ be the ring of $A$-endomorphisms of $W$ which is generated by the scalar multiplications with elements of $C$ and the $y_{i}$ 's. We have $y_{i} y_{j}=y_{j} y_{i}$, $y_{i}^{p}=y_{i}+\alpha_{i}$ and for $c \in C, y_{i} c=c y_{i}+\left(\partial_{i} c\right)$. Furthermore, the monomials $y_{1}^{e_{1}} \cdots y_{r}^{e_{r}}$ with $0 \leqq e_{i}<p$ are a $C$-module basis for $R$. Now every element of $\mathfrak{I}$ can be written uniquely in the form $s=c_{0}+\sum_{i=1}^{r} c_{i} s_{i}$ with $c_{i} \in C$. We define $\zeta(s)=c_{0}+\sum_{i=1}^{r} c_{i} y_{i} \in R$. If we regard $R$ as a restricted Lie algebra in the usual way, we see at once that $\zeta: \mathfrak{l} \rightarrow R$ is a restricted Lie algebra monomorphism. Hence $\zeta$ can be extended uniquely to a homomorphism of $I(\mathfrak{l})$ onto $R$ which we shall still denote by $\zeta$. Evidently $\zeta$ maps the ideal $J$ of $I(\mathfrak{l})$ to 0 , and since it maps $\mathfrak{I}$ monomorphically we have $J \cap \mathfrak{l}=0$. Hence we may identify $\mathfrak{l}$ with its canonical image in $V_{L}$. Furthermore, $\zeta$ induces a homomorphism $\gamma$ of $V_{L}$ onto $R$ which (with the identification we have made) leaves the elements of $C$ fixed. Since the cosets modulo $J$ of the ordered monomials in the $s_{i}$ with nonnegative exponents less than $p$ form a basis for $V_{L}$ over $C, \gamma: V_{L} \rightarrow R$ is an isomorphism. Hence we may verify the remaining assertions concerning $V_{L}$ by operating on $R$.

We write the elements of $R$ as polynomials in the $y_{i}$ with coefficients in $C$, the degree in each $y_{i}$ being at most $p-1$. We claim that if $x \in R$ and $x u_{i}-u_{i} x \in C$, then the degree of $x$ in $y_{i}$ is at most 1 . In fact, write $x=x_{0}+x_{1} y_{i}+\cdots+x_{m} y_{i}^{m}$ where $x_{j}$ do not contain $y_{i}$ and $m<p$. Then from $y_{i} u_{i}=u_{i}\left(1+y_{i}\right), y_{i}^{j} u_{i}=u_{i}\left(1+y_{i}\right)^{j}$, we find

$$
\begin{aligned}
x u_{i}-u_{i} x & =\sum_{j=0}^{m}\left(x_{j} y_{i}^{j} u_{i}-u_{i} x_{j} y_{i}^{j}\right)=\sum_{j=0}^{m} x_{j}\left(y_{i}^{j} u_{i}-u_{i} y_{i}^{j}\right) \\
& =\sum_{j=0}^{m} x_{j} u_{i}\left(\left(1+y_{i}\right)^{j}-y_{i}^{j}\right)=m x_{m} u_{i} y_{i}^{m-1}+\sum_{j=0}^{m-2} b_{j} y_{i}^{j}
\end{aligned}
$$

where $b_{j}$ do not contain $y_{i}$. This shows that our condition on $x$ implies that $x_{m}=0$ when $m>1$, and thus establishes our claim. By a similar computation, it is easy to show that if $x \in R$ and $x u_{i}-u_{i} x \in C$ for all $i=1, \ldots, r$, then $x$ must be of the form 
$c_{0}+\sum_{i=1}^{r} c_{i} y_{i}$ with $c_{\imath} \in C$. So $\mathfrak{I}$ is precisely the set of all $x \in V_{L}$ for which $x c-c x \in C$ for all $c$ in $C$. We conclude also that the center of $V_{L}$ is contained in $\mathfrak{I}$ and since the only elements of $\mathfrak{I}$ which commutes with all elements of $C$ are the elements of $C$, we find that the center of $V_{\dot{L}}$ is contained in $C$ and hence it coincides with $A$. Now let $I$ be any nonzero two-sided ideal of $\bar{R}=R / \mathfrak{q} R$ where $\mathfrak{q}$ is the maximal ideal of $A$ and let $\bar{x}$ be a nonzero element of $I$. Write $\bar{u}_{i}=u_{i}+\mathfrak{q} R$ and compute $\bar{x} \bar{u}_{i}-\bar{u}_{i} \bar{x}$ as above. Our computation shows that if we repeat this a suitable number of times and with suitable indices $i$, we finally obtain a nonzero element of $I \cap(C / q C)$. But the ideal $I \cap(C / \mathfrak{q} C)$ is stable under $\mathfrak{g} / \mathfrak{q} g$. Since $C[\mathfrak{g}]=\operatorname{End}_{A}(C)$ implies $(C / \mathfrak{q} C)[\mathfrak{g} / \mathfrak{q} g]=\operatorname{End}_{A / \mathfrak{q}}(C / \mathfrak{q} C)$ which in turn implies no nontrivial ideal in $C / \mathfrak{q} C$ is stable under $\mathfrak{g} / \mathfrak{q} g$, we must have $I \cap(C / \mathfrak{q} C)=C / \mathfrak{q} C$. So $I=\bar{R}$. This shows $R$ is central separable over $A$ because $\bar{R}$ is central simple over $A / \mathfrak{q}[5$, p. 180, Exercise 14]. It follows from our construction of $V_{L}$ that the inner derivation effected by an element $s$ in $\mathfrak{l}$ coincides with $\varphi(s)$ on $C$, and hence $L$ is indeed the regular extension which is derived from the central separable $A$-algebra $V_{L}$ with the splitting subalgebra $C$.

There remains to prove the last part of the theorem. In the notation used there it is clear that the canonical homomorphism of $I(\mathfrak{l})$ into $R$ annihilates the ideal $J$ and hence induces a homomorphism $\gamma$ of $V_{L}$ into $R$. This homomorphism $\gamma$ leaves the elements of $C$ fixed and in particular is not 0 . Since $V_{L}$ is central separable, $\gamma$ therefore must be monomorphic. Regarding $R$ as a two-sided $V_{L}$-module via $\gamma$, we have by [2, Theorem 3.1] $R \cong V_{L} \otimes_{A} R^{\gamma\left(V_{L}\right)}=V_{L}$ because $R^{\gamma\left(V_{L}\right)}=\{x \in R \mid x y=y x$ for all $\left.y \in \gamma\left(V_{L}\right)\right\}$ is just $A$.

REMARK. The above proof for Theorem 2 is adapted from [11, pp. 484-485] with only minor changes.

Let $R$ and $S$ be two central separable $A$-algebras both with $C$ as a splitting subalgebra. We say $R$ is equivalent to $S$ if and only if there is an $A$-algebra isomorphism $\alpha: R \rightarrow S$ such that $\alpha(c)=c$ for all $c$ in $C$. Let $\mathscr{A}(C / A)$ denote the set of all equivalence classes of such algebras. Chase-Rosenberg [8, Theorem 2.14] show that $\mathscr{A}(C / A)$ form an abelian group with respect to the product $R \cdot S=$ $\operatorname{End}_{R \otimes S}\left(R \otimes_{C} S\right)$ where $R \otimes_{C} S$ is regarded as a right $(R \otimes S)$-module, $R$ and $S$ are regarded as left $C$-modules (i.e., $\left.R \otimes_{C} S=\left(R \otimes_{C} S\right)_{R \otimes S}, R={ }_{C} R, S={ }_{C} S\right)$.

THEOREM 3. The map $\nu: \mathscr{E}(\mathrm{g}, C) \rightarrow \mathscr{A}(C / A), L \rightarrow V_{L}$, is an isomorphism of groups.

Proof. It follows from Theorem 2 and Lemma 6 that $\nu$ is an isomorphism of sets. So it suffices to prove that $\nu$ is a group-homomorphism. We interrupt the proof with a lemma.

LEMMA 10. Let $R$ and $S$ be central separable A-algebras both with $C$ as a splitting subalgebra. Then the map from $R \cdot S \equiv \operatorname{End}_{R \otimes S}\left(R \otimes_{C} S\right)$ into

$$
T \equiv\left\{\sum u_{i} \otimes u_{i} \in R \otimes_{C} S \mid \sum u_{i} c \otimes v_{i}=\sum u_{i} \otimes v_{i} c \text { for all } c \text { in } C\right\}
$$

given by $f \rightarrow f(1)$ is an A-algebra isomorphism which leaves $C$ pointwise fixed. 
Proof. It is clear that given $f$ in $R \cdot S, f$ is determined by its action on 1 . Write $f(1)=\sum u_{i} \otimes v_{i}$. Then $\sum u_{i} c \otimes v_{i}=\sum u_{i} \otimes v_{i} c$, for all $c \in C$, because in $R \otimes_{C} S$, $c \otimes 1=1 \otimes c$. It is also easily verified that given any element $\sum u_{i} \otimes v_{i}$ in $R \otimes_{C} S$ such that $\sum u_{i} c \otimes v_{i}=\sum u_{i} \otimes v_{i} c$ for all $c$ in $C$, then the left multiplication by $\sum u_{i} \otimes v_{i}, x \otimes y \rightarrow \sum u_{i} x \otimes v_{i} y$, is a well-defined map on $R \otimes_{C} S$ and belongs to $R \cdot S$. This completes the proof of the lemma.

Now return to the proof of Theorem 3. Let $L: 0 \rightarrow C \rightarrow \mathfrak{l} \stackrel{\oplus}{\rightarrow} \mathfrak{g} \rightarrow 0$ and $L^{\prime}$ : $0 \rightarrow C \leftrightarrow \stackrel{\mathfrak{l}^{\prime}}{\varphi^{\prime}} \rightarrow \mathrm{g} \rightarrow 0$ be regular extensions. We would like to show that the regular extension $H: 0 \rightarrow C \hookrightarrow \mathfrak{h} \rightarrow \mathfrak{g} \rightarrow 0$ associated to

$$
T=\left\{\sum u_{i} \otimes v_{i} \in V_{L} \otimes_{C} V_{L^{\prime}} \mid \sum u_{i} c \otimes v_{i}=\sum u_{i} \otimes v_{i} c \text { for all } c \text { in } C\right\}
$$

is equivalent to $L+L^{\prime}: 0 \rightarrow C \rightarrow \mathfrak{t} \rightarrow \mathfrak{g} \rightarrow 0$. By Lemma 10 and Theorem 2 , this will prove that $\nu$ is a group-homomorphism $\left({ }^{2}\right)$.

We have the following diagram

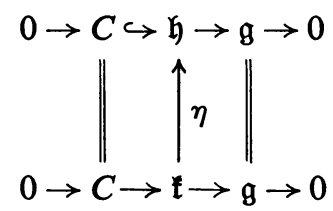

where $\eta$ is the map $\left(s, s^{\prime}\right) \rightarrow s \otimes 1+1 \otimes s^{\prime}$. To see $\eta$ is well defined, we first note that for all $c$ in $C$

$$
\begin{aligned}
\left(s c \otimes 1+c \otimes s^{\prime}\right)-\left(s \otimes c+1 \otimes s^{\prime} c\right) & =(s c-c s) \otimes 1+1 \otimes\left(c s^{\prime}-s^{\prime} c\right) \\
& =[s, c] \otimes 1-1 \otimes\left[s^{\prime}, c\right]=0
\end{aligned}
$$

because $[s, c]=\varphi(s)(c)=\varphi^{\prime}\left(s^{\prime}\right)(c)=\left[s^{\prime}, c\right]$. This shows $\eta\left(s, s^{\prime}\right)$ is an element of $T$. Now

$\left(s \otimes 1+1 \otimes s^{\prime}\right)(c \otimes 1)-(c \otimes 1)\left(s \otimes 1+1 \otimes s^{\prime}\right)=(s c-c s) \otimes 1=[s, c] \otimes 1 \in C$

for all $c$ in $C$. So $\eta\left(s, s^{\prime}\right)$ is actually in $\mathfrak{h}$. The last computation also shows that the right-hand side square in the above diagram is commutative. That the left-hand side square is commutative is of course clear. It is also readily verified that $\eta$ is a $C$-linear restricted Lie ring-homomorphism. By the five lemma, $\eta$ must be an isomorphism.

REMARK. Theorem 3 may be regarded as a generalization of [11, Theorem 6] because when $C$ is a field, $\mathscr{A}(C / A)$ is exactly the Brauer group $B(C / A)$ [8, Theorem 2.14].

3. Differentials. In order to produce a map from the Amitsur cohomology group $H^{2}\left(C / A, G_{m}\right)$ into the group $\mathscr{E}(g, C)$, it is necessary to put our earlier work

$\left({ }^{2}\right)$ We are indebted to the referee for the following argument which replaces a lengthier argument used in the original proof. 
[15] on logarithmic derivatives in an invariant form. The following exposition on differentials is based on that of Cartier's [7, pp. 187-204]. The proof for Lemma 14 is taken from [15, Theorem 2.6].

We shall write $\Omega^{1}=\operatorname{Hom}_{C}(\mathfrak{g}, C)$, and denote by $\Omega^{i}$ the $i$ th exterior product $\Omega^{1} \wedge \cdots \wedge \Omega^{1}$ ( $i$ factors). The exterior algebra $\Omega$ of $\Omega^{1}$ over $C$ is the direct sum $C+\Omega^{1}+\Omega^{2}+\cdots$. It follows from [16, Theorem 10] that for any multiplicatively closed subset $S$ of $A, \mathfrak{g}_{S}$ may be identified with the Lie algebra $\mathrm{g}\left(C_{S} / A_{S}\right)$ of all $A_{S}$-derivations on $C_{S}$. Consequently $\Omega_{S}$ may be identified with the exterior algebra of Hom $C_{S}\left(\mathrm{~g}\left(C_{S} / A_{S}\right), C_{S}\right)$ over $C_{S}$. For any $x$ in $C$, we shall denote by $d x$ the map $\mathrm{g} \rightarrow C$, given by $\partial \rightarrow \partial x$. The map $d: C \rightarrow \Omega^{1}, x \rightarrow d x$, is $A$-linear and we have the identity

$$
d(x y)=x d y+y d x, \quad x, y \in C .
$$

LEMMA 11. There is a unique A-linear map $d: \Omega \rightarrow \Omega$ called the exterior differentiation on $\Omega$ which satisfies the following conditions:

(i) $d\left(\omega \wedge \omega^{\prime}\right)=d \omega \wedge \omega^{\prime}+(-1)^{i} \omega \wedge d \omega^{\prime}\left(\omega \in \Omega^{i}, \omega^{\prime} \in \Omega\right)$,

(ii) $d(d \omega)=0(\omega \in \Omega)$,

(iii) $d$ is the extension of the map $C \rightarrow \Omega^{1}, x \rightarrow d x$.

Proof. We shall first prove the uniqueness of $d$. Assume there are two such maps $d_{1}, d_{2}$. Let $\Upsilon$ be the set of all $\omega$ in $\Omega$ such that $d_{1} \omega=d_{2} \omega$. By (iii), we have $C \subset \Upsilon$. By (i), $\Upsilon$ is a subalgebra of $\Omega$. Since $\Omega$ as a $C$-algebra is generated by the elements of $\Omega^{1}$, the uniqueness of $d$ would follow if we could show that $\Omega^{1}$ is contained in $\Upsilon$. By (ii), it is clear that the $C$-submodule $C(d C)$ of $\Omega^{1}$ generated by the image of $d: C \rightarrow \Omega^{1}$ is contained in $\Upsilon$. We claim that the inclusion map $C(d C) \hookrightarrow \Omega^{1}$ is onto. It suffices of course to prove this is the case when $A$ is local. But when $A$ is local, $C$ admits a $p$-basis $x_{1}, \ldots, x_{r}$ over $A$, and it is readily verified that the $d x_{i}$ form a $C$-module basis of $\Omega^{1}$. This proves the uniqueness of $d$.

There remains to show the existence of the exterior differentiation on $\Omega$. Assume first that $C$ admits a $p$-basis $x_{1}, \ldots, x_{r}$ over $A$ so that the elements of the form $d x_{i_{1}} \wedge \cdots \wedge d x_{i_{s}}, i_{1}<\cdots<i_{s}, 0 \leqq s \leqq r$, constitute a $C$-module basis for $\Omega$. We therefore have a map

$$
\begin{aligned}
\Omega & \rightarrow \Omega, \\
x \cdot d x_{i_{1}} \wedge \cdots \wedge d x_{i_{s}} & \rightarrow d x \wedge d x_{i_{1}} \wedge \cdots \wedge d x_{i_{s}} \quad(x \in C) .
\end{aligned}
$$

It is a mechanical matter to verify that this map satisfies all the requirements of our lemma. We shall therefore not go into the details here.

In the general case, let $\widetilde{\Omega}$ be the associated sheaf of the $A$-module $\Omega$. For each $\mathfrak{q}$ in spec $A$, the fibre of $\Omega$ at $\mathfrak{q}$ is just $\Omega_{\mathfrak{q}}$. Since $C_{\mathfrak{q}}$ admits a $p$-basis over $A_{\mathfrak{q}}$, there exists an exterior differentiation $d_{\mathfrak{q}}$ on $\Omega_{\mathrm{q}}$. Let $\tilde{d}: \Omega \rightarrow \Omega$ be the (set-theoretical) map such that the restriction of $\tilde{d}$ to $\Omega_{q}$ coincides with $d_{\mathfrak{q}}$. To see $\tilde{d}$ is continuous we recall that for any $q$ in $\operatorname{Spec} A$, there exists some $f \in A-q$ such that $C_{f}$ admits a 
$p$-basis over $A_{f}\left[16\right.$, Lemma 7]. So there exists an exterior differentiation $d_{f}$ on $\Omega_{f}$. It follows from the uniqueness of the exterior differentiation that the diagram

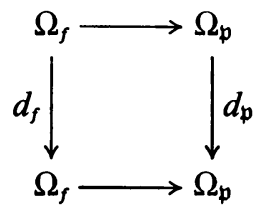

is commutative for every $\mathfrak{p}$ in the open set $D_{f}=\{\mathfrak{p} \in \operatorname{Spec} A \mid f \notin \mathfrak{p}\}$. Let $\tilde{d}_{f}$ be the endomorphism on the $\tilde{A}_{f}$-module $\widetilde{\Omega}_{f}$ induced by $d_{f}$. Since $\tilde{\Omega}_{f}$ is just the restriction of $\widetilde{\Omega}$ to $D_{f}\left[9\right.$, p. 86, Proposition (1.3.6)] and the restriction of $\tilde{d}$ to $\tilde{\Omega}_{f}$ coincides with $\tilde{d}_{f}, \tilde{d}$ must be continuous. If we denote by $d$ the $A$-module endomorphism on $\Omega$ associated to $\tilde{d}\left[9\right.$, p. 87 , Corollary (1.3.8)], we see that $d^{2}$ is zero because $\tilde{d}^{2}$ is. For any $\omega$ in $\Omega^{i}$ and $\omega^{\prime}$ in $\Omega$, the difference $d\left(\omega \wedge \omega^{\prime}\right)-\left(d \omega \wedge \omega^{\prime}+(-1)^{i} \omega \wedge d \omega^{\prime}\right)$ is zero because it is zero at every $q$ in Spec $A$. Finally the restriction of $d$ to $C$ coincides with the map $x \rightarrow d x, x \in C$, because they coincide locally. This completes the proof of the existence of the exterior differentiation on $\Omega$.

LEMMA 12. If $x$ is an element of $C$, then $x^{p-1}\left(\partial^{p} x\right)-\partial^{p-1}\left(x^{p-1}(\partial x)\right)=(\partial x)^{p}$ for any derivation $\partial$ on $C$.

Proof. Let $P$ denote the (prime) field of $p$ elements. Let $K$ be the polynomial ring $P\left[t_{0}, t_{1}, t_{2}, \ldots\right]$ where the $t_{i}$ 's are independent variables. We have a homomorphism $\theta: K \rightarrow C$ which is given by $\theta\left(t_{0}\right)=x$ and $\theta\left(t_{i}\right)=\partial^{i} x, i>0$. If we denote by $\Delta$ the derivation on $K$ given by $\Delta t_{i}=t_{i+1}$, we have the following commutative diagram

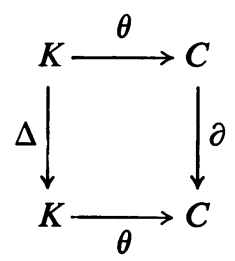

Now $\Delta$ has a unique extension to the field $L$ of fractions of $K$, which we again denote by $\Delta$. Let $\Delta+\Lambda\left(t_{0}^{-1} t_{1}\right): L \rightarrow L$ be the map $s \rightarrow \Delta(s)+t_{0}^{-1} t_{1} s$. We have

$$
\begin{aligned}
\Lambda\left[\left(t_{0}^{-1} t_{1}\right)^{p}+\Delta^{p-1}\left(t_{0}^{-1} t_{1}\right)-t_{0}^{-1} t_{p}\right] & \\
= & \left(\Lambda\left[\left(t_{0}^{-1} t_{1}\right)^{p}+\Delta^{p-1}\left(t_{0}^{-1} t_{1}\right)\right]+\Delta^{p}\right)-\left(\Delta^{p}+\Lambda\left(t_{0}^{-1} t_{p}\right)\right) \\
& =\left(\Lambda\left(t_{0}^{-1} t_{1}\right)+\Delta\right)^{p}-\left(\Delta^{p}+\Lambda\left(t_{0}^{-1}\left[\Delta^{p} t_{0}\right]\right)\right) .
\end{aligned}
$$

The second equality follows from Jacobson's formula [7, p. 201, (36)]. From $\Delta+\Lambda\left(t_{0}^{-1} t_{1}\right)=\left(\Lambda t_{0}^{-1}\right) \Delta\left(\Lambda t_{0}\right)$ we see that $\left(\Delta+\Lambda\left(t_{0}^{-1} t_{1}\right)\right)^{p}=\left(\Lambda t_{0}^{-1}\right) \Delta^{p}\left(\Lambda t_{0}\right)=\Delta^{p}$ $+\Lambda\left(t_{0}^{-1}\left[\Delta^{p} t_{0}\right]\right)$. So $\left(t_{0}^{-1}\left(\Delta t_{0}\right)\right)^{p}+\Delta^{p-1}\left(t_{0}^{-1}\left(\Delta t_{0}\right)\right)-t_{0}^{-1}\left(\Delta^{p} t_{0}\right)=0$. Clearing the denominators we get $\left(\Delta t_{0}\right)^{p}+\Delta^{p-1}\left(t_{0}^{p-1}\left(\Delta t_{0}\right)\right)-t_{0}^{p-1}\left(\Delta^{p} t_{0}\right)=0$. Applying $\theta$ to the equation, we get $(\partial x)^{p}+\partial^{p-1}\left(x^{p-1}(\partial x)\right)-x^{p-1}\left(\partial^{p} x\right)=0$ because $\theta \Delta^{i}=\partial^{i} \theta$ for any $i$. This completes the proof of the lemma. 
By formula (i) of Lemma 11, the kernel $Z$ of $d: \Omega \rightarrow \Omega$ is an $A$-subalgebra of $\Omega$ while the image $B$ of $d$ is an ideal in $Z$. We have the following:

Lemma 13. The A-subalgebra $Z$ is equal to the sum of $B$ and the $A$-subalgebra $R$ of $\Omega$ generated by elements of the form $x^{p-1} d x$ with $x$ in $C$.

Proof. We have the obvious inclusion $R+B \hookrightarrow Z$. To show the map is onto, we may assume $A$ is a local ring. So $C$ admits a $p$-basis $x_{1}, \ldots, x_{r}$ over $A$. In this situation, a slightly more specific statement holds: $Z$ is the direct sum of $B$ and the $A$-subalgebra of $\Omega$ generated by the elements $x_{i}^{p-1} d x_{i}$. This statement appears in [7, p. 197, Proposition 6] where the proof is given in the case where $C$ is a field. But the computations are formal and are equally valid in our case.

Now let $\Xi$ denote the kernel of $d: \Omega^{1} \rightarrow \Omega^{2}$. Given $\omega$ in $\Xi$ we have a map

$$
\begin{aligned}
\Gamma \omega: \mathfrak{g} & \rightarrow A, \\
\partial & \rightarrow \omega\left(\partial^{p}\right)-\partial^{p-1}(\omega(\partial))
\end{aligned}
$$

$\Gamma$ is called the Cartier operator. It is not obvious that $(\Gamma \omega)(\partial)$ actually belongs to $A$. By Lemma 13, we may write $\omega=d u+\sum \alpha_{i} u_{i}^{p-1} d u_{i}, u, u_{i} \in C, \alpha_{i} \in A$. Since $\Gamma$ is $A$-linear and $\Gamma(d u)=0$, it suffices to show that $\left(\Gamma x^{p-1} d x\right)(\partial)=x^{p-1}\left(\partial^{p} x\right)-$ $\partial^{p-1}\left(x^{p-1} \partial x\right), x \in C, \partial \in \mathfrak{g}$, is an element of $A$. This, however, is a simple consequence of Lemma 12. Now $A$ may be regarded as a $C$-module via the scalar product $C \times A$ $\rightarrow A,(c, a) \rightarrow c^{p} a$. We wish to show that $\Gamma \omega$ is an element of $\operatorname{Hom}_{C}(\mathrm{~g}, A)$. It suffices to verify the special case $\omega=x^{p-1} d x$, of course. Using Lemma 12 again, we find $\left(\Gamma x^{p-1} d x\right)(c \partial)=((c \partial) x)^{p}=c^{p}(\partial x)^{p}=c^{p}\left(\Gamma x^{p-1} d x\right)(\partial)$ and

$$
\begin{aligned}
\left(\Gamma x^{p-1} d x\right)\left(\partial+\partial^{\prime}\right) & =\left(\left(\partial+\partial^{\prime}\right) x\right)^{p}=(\partial x)^{p}+\left(\partial^{\prime} x\right)^{p} \\
& =\left(\Gamma x^{p-1} d x\right)(\partial)+\left(\Gamma x^{p-1} d x\right)\left(\partial^{\prime}\right)
\end{aligned}
$$

as desired. Summarizing, we see that $\Gamma: \Xi \rightarrow \operatorname{Hom}_{C}(\mathfrak{g}, A)$ is an additive group homomorphism. Therefore the map

$$
\delta_{1}: \Xi \rightarrow \operatorname{Hom}_{C}(\mathfrak{g}, A)
$$

given by $\left(\delta_{1} \omega\right)(\partial)=(\Gamma \omega)(\partial)-(\omega(\partial))^{p}, \partial \in \mathfrak{g}$, is also an additive group homomorphism.

Now let $\delta_{0}: C^{*} \rightarrow \Xi$ be the map $x \rightarrow x^{-1} d x$. It is readily verified that $\delta_{1} \delta_{0}=0$. So we have a complex

$$
0 \longrightarrow A^{*} \longrightarrow C^{*} \stackrel{\delta_{0}}{\longrightarrow} \Xi \stackrel{\delta_{1}}{\longrightarrow} \operatorname{Hom}_{C}(\mathrm{~g}, A) .
$$

LEMMA 14. The group $L(C / A)=\left(\right.$ kernel $\left.\delta_{1}\right) /\left(\right.$ image $\left.\delta_{0}\right)$ is isomorphic to the rank one projective class group $P(C / A)$.

Proof. Let $M$ be a rank one projective $A$-module such that the $C$-module $M \otimes C$ is free on one generator $b$. Let $\mathfrak{p}$ be a prime ideal in $A$. To any generator $b_{\mathfrak{p}}$ for the free $A_{\mathfrak{p}}$-module $M \otimes A_{\mathfrak{p}}$ there is a unique invertible element $u_{\mathfrak{p}}$ in $C_{\mathfrak{p}}$ such that the equation $b=b_{\mathfrak{p}}\left(1 \otimes u_{\mathfrak{p}}\right)$ holds in $M \otimes C_{\mathfrak{p}}$. If $b_{\mathfrak{p}}^{\prime}$ is another generator for $M \otimes A_{\mathfrak{p}}$ 
and $u_{\mathfrak{p}}^{\prime}$ the corresponding invertible element in $C_{\mathfrak{p}}$, then $u_{\mathfrak{p}}^{\prime} u_{\mathfrak{p}}^{-1}$ is in $A_{\mathfrak{p}}$. This shows the correspondence $s: \mathfrak{p} \rightarrow u_{\mathfrak{p}}^{-1} d u_{\mathfrak{p}}$ is independent of the choice of $b_{\mathfrak{p}}$. To see $s$ is a section for the sheaf $\widetilde{\Omega}^{1}$, we note that for any $\mathfrak{p}$ in Spec $A$, there is some $f \in A-\mathfrak{p}$ such that the $A_{f}$-module $M \otimes A_{f}$ is free on one generator $b_{f}$. So there is an invertible element $u_{f}$ in $C_{f}$ such that the equation $b=b_{f}\left(1 \otimes u_{f}\right)$ holds in $M \otimes C_{f}$. It is clear that for any $q$ in the distinguished open set $D_{f}, u_{f}^{-1} d u_{f}$ goes to $u_{\mathfrak{q}}^{-1} d u_{\mathfrak{q}}$ under the canonical map $\Omega_{f}^{1} \rightarrow \Omega_{\mathrm{q}}^{1}$. So $s$ is a section for $\widetilde{\Omega}^{1}$ and there is a unique element $\omega \in \Omega^{1}$ such that for all $\mathfrak{p}$ in Spec $A$, the canonical image of $\omega$ in $\Omega_{\mathfrak{p}}^{1}$ is $u_{\mathfrak{p}}^{-1} d u_{\mathfrak{p}}$. Now both $d \omega$ and $\delta_{1} \omega$ must be zero because locally they are zero. So $\omega$ is actually in $\Xi$. If $b^{\prime}$ is another generator for the free $C$-module $M \otimes C$ and $\omega^{\prime}$ is the element in $\Xi$ to correspond, then $\omega=\omega^{\prime}$ modulo image $\delta_{0}$. So we have a well-defined map $\theta: P(C / A) \rightarrow L(C / A)$. It is readily verified that $\theta$ is a group-homomorphism. To show it is one to one, assume $\omega=x^{-1} d x$ for some $x \in C^{*}$. Then for any $\mathfrak{p}$ in $\operatorname{Spec} A$, the equation $x^{-1} d x=u_{\mathfrak{p}}^{-1} d u_{\mathfrak{p}}$ holds in $\Omega_{\mathfrak{p}}^{1}$. We have, for any $A_{\mathfrak{p}}$-derivation $\partial$ on $C_{\mathfrak{p}}$, $\partial\left(u_{\mathfrak{p}} x^{-1}\right)=\left(u_{\mathfrak{p}} x^{-1}\right)\left(u_{\mathfrak{p}}^{-1} \cdot \partial u_{\mathfrak{p}}-x^{-1} \cdot \partial x\right)=0$ which implies $u_{\mathfrak{p}}=x \alpha_{\mathfrak{p}}$ for some $\alpha_{\mathfrak{p}}$ in $A_{\mathfrak{p}}^{*}$ because $A_{\mathfrak{p}}$ is exactly the set of all elements of $C_{\mathfrak{p}}$ which are annihilated by every $A_{\mathfrak{p}}$-derivation on $C_{\mathfrak{p}}$ [16, Theorem 9]. It follows that $(1 \otimes \partial)\left(b\left[1 \otimes x^{-1}\right]\right), \partial \in \mathfrak{g}$, must be zero because at every $\mathfrak{p},(1 \otimes \partial)\left(b\left[1 \otimes x^{-1}\right]\right)=(1 \otimes \partial)\left(b_{\mathfrak{p}}\left[1 \otimes \alpha_{\mathfrak{p}}\right]\right)=0$. Since the sequence

$$
0 \longrightarrow M \otimes A \longrightarrow M \otimes C \underset{\{1 \oplus \partial\}}{\longrightarrow} \sum M \otimes C \text { (direct sum) }
$$

is exact, $b\left(1 \otimes x^{-1}\right)$ is therefore an element of $M$. If $m$ is any element of $M$, then $m \otimes 1=b\left(1 \otimes x^{-1} c\right)$ for some $c \in C$. Now $c$ must be an element of $A$ because $b\left(1 \otimes x^{-1}(\partial c)\right)=(1 \otimes \partial)(m \otimes 1)=0$ for all $\partial \in \mathrm{g}$. This shows $M$ is a free $A$-module based on $b\left(1 \otimes x^{-1}\right)$, whence $\theta$ is one to one.

There remains to show that $\theta$ is onto. So let $\omega \in \Xi$ be an element in the kernel of $\delta_{1}$. Let $L$ be the trivial regular extension $0 \rightarrow C \hookrightarrow C+g \rightarrow g \rightarrow 0$. By Theorem 3, $L$ is the regular extension derived from the central separable $A$-algebra $\operatorname{End}_{A}(C)$. We have a map

$$
\begin{aligned}
\mathfrak{x}: C+\mathfrak{g} & \rightarrow \operatorname{End}_{A}(C), \\
c+\partial & \rightarrow \partial+\Lambda(c+\omega(\partial)),
\end{aligned}
$$

where $\Lambda x, x \in C$, is the map on $C$ produced by multiplication by $x$. The assumption that $d \omega=0, \delta_{1} \omega=0$ says that $\mathfrak{r}$ is a restricted Lie algebra homomorphism [7, pp. 200-201]. One verifies at once that $\mathfrak{r}$ induces an $A$-algebra homomorphism $\rho: V_{L} \rightarrow \operatorname{End}_{A}(C)$. By Theorem 2, the inclusion map $C+\mathfrak{g} \hookrightarrow \operatorname{End}_{A}(C)$ induces an $A$-algebra isomorphism $\gamma: V_{L} \rightarrow \operatorname{End}_{A}(C)$. So the additive group $C^{+}$has a new $\operatorname{End}_{A}(C)$-module structure via the scalar product $\operatorname{End}_{A}(C) \times C \rightarrow C,(f, x) \rightarrow$ $\left(\rho \gamma^{-1}(f)\right)(x)$. Write this new module as ${ }_{\omega} C$. By [5, p. 181, Exercise 18], we have $\operatorname{Hom}_{E}\left(C,{ }_{\omega} C\right) \otimes C \cong{ }_{\omega} C$, where $E=\operatorname{End}_{A}(C)$. It follows from [5, p. 53] that $\operatorname{Hom}_{E}\left(C,{ }_{\omega} C\right)$ is a rank one projective $A$-module. Now each element of $\operatorname{Hom}_{E}\left(C,{ }_{\omega} C\right)$ 
is determined by its action on $1 \in C$ which must go to an element of ${ }_{\omega} C$ annihilated by the new operation of $g$ since in the old operation of $\mathfrak{g}, \partial 1=0$ for all $\partial \in \mathfrak{g}$. Thus $\operatorname{Hom}_{E}\left(C,{ }_{\omega} C\right) \cong \bigcap\{$ kernel $\partial+\Lambda(\omega \partial) \mid \partial \in \mathfrak{g}\}$. Write $M=\bigcap\{$ kernel $\partial+\Lambda(\omega \partial) \mid \partial \in \mathfrak{g}\}$ and let $b$ be the element $\sum m_{i} \otimes c_{i}$ in $M \otimes C$ such that $\sum m_{i} c_{i}=1$ in $C$. For each $\mathfrak{p} \in \operatorname{Spec} A$, pick $m_{\mathfrak{p}} \in M$ such that $b_{\mathfrak{p}}=m_{\mathfrak{p}} \otimes 1$ is a generator for the one dimensional free $A_{\mathfrak{p}}$-module $M_{\mathfrak{p}}$. We have, for all $i, m_{\mathfrak{i}} \otimes 1=m_{\mathfrak{p}} \otimes a_{\mathfrak{i}}$ for some $a_{\mathfrak{i}} \in A_{\mathfrak{p}}$. Now with the notations introduced earlier in the proof, $u_{\mathfrak{p}}=\sum a_{\mathfrak{i}} c_{\mathfrak{i}}$. But in $C_{\mathfrak{p}}$, $m_{\mathfrak{p}} u_{\mathfrak{p}}=m_{\mathfrak{p}} \sum a_{\mathfrak{i}} c_{\mathfrak{i}}=\sum m_{\mathfrak{i}} c_{\mathfrak{i}}=1$. So $0=u_{\mathfrak{p}} \cdot d m_{\mathfrak{p}}+m_{\mathfrak{p}} \cdot d u_{\mathfrak{p}}=-u_{\mathfrak{p}} m_{\mathfrak{p}} \omega+m_{\mathfrak{p}} d u_{\mathfrak{p}}=-\omega+$ $u_{\mathfrak{p}}^{-1} d u_{\mathfrak{p}}$ whence $\theta$ is onto. This completes the proof of the lemma.

Remarks. (1) The $A$-module $\Xi$ is finitely generated projective. For if $f$ is an element of $A$ such that $C_{f}$ admits a $p$-basis over $A_{f}$, then $\Xi_{f}$ is a free $A_{f}$-module of dimension $p^{r}+r-1$ where $p^{r}=\left[C_{f}: A_{f}\right]$.

(2) If $C$ is obtained from $A$ by adjoining nilpotents, then $L(C / A)=0$. For any $\omega$ in kernel $\delta_{1}$, we have $\sum m_{i} c_{i}=1$ for some $m_{i}$ in $M=\bigcap\{\operatorname{kernel}(\partial+\Lambda(\omega \partial)) \mid \partial \in \mathfrak{g}\}$ and $c_{i}$ in $C$. Pick $f_{i}$ in $A$ such that $c_{i}-f_{i}$ is nilpotent in $C$. Then $\sum m_{i} f_{i} \in M$ as the sum of 1 and a nilpotent $\sum m_{i}\left(f_{i}-c_{i}\right)$ must be a unit in $C$. Write $x^{-1}=\sum m_{i} f_{i}$. One sees at once that $\omega=x^{-1} d x$.

(3) It will be shown later in $\S 5$ that the cokernel of $\delta_{1}: \Xi \rightarrow \operatorname{Hom}_{C}(\mathfrak{g}, A)$ is isomorphic to $\mathscr{E}(g, C)$.

LEMma 15. Let $\omega$ be an element of $\Omega^{1}$. Then $\omega$ is equal to $d x$ for some $x$ in $C$ if and only if $d \omega=0$ and $\Gamma \omega=0$.

Proof. If $\omega=d x$ for some $x$ in $C$, then of course $d \omega=0$ and $\Gamma \omega=0$. So it suffices to show that $\omega$ must be of the form $d x$ whenever both $d \omega$ and $\Gamma \omega$ are zero. Let $B$ denote the image of $d: C \rightarrow \Omega^{1}$. Let $B^{\prime}$ be the $A$-submodule $B+A \omega$ of $\Omega^{1}$. Now the inclusion map $\iota: B \rightarrow B^{\prime}$ is onto if and only if it is onto at every $\mathfrak{p} \in \operatorname{Spec} A$. We may therefore assume that $C$ admits a $p$-basis $x_{1}, \ldots, x_{r}$ over $A$ so that $\omega$ is of the form $\alpha_{1} x_{1}^{p-1} d x_{1}+\cdots+\alpha_{r} x_{r}^{p-1} d x_{r}+d x$ for some $\alpha_{i}$ in $A$ and $x$ in $C$. For any $\partial$ in $\mathfrak{g}$, we have

$$
0=(\Gamma \omega)(\partial)=\sum_{i=1}^{r} \alpha_{i}\left(\Gamma x_{i}^{p-1} d x_{i}\right)(\partial)=\sum_{i=1}^{r} \alpha_{i}\left(\partial x_{i}\right)^{p} .
$$

In this equation, if we let $\partial$ be the $A$-derivation on $C$ given by $\partial x_{j}=\delta_{i j}$, we see that $\alpha_{i}=0$. This completes the proof of the lemma.

4. Applications to Amitsur cohomology. Let $R$ be a commutative $A$-algebra such that $R$ is flat as an $A$-module. Since $\mathfrak{g}$ is finitely generated projective as a $C$-module, we have $\operatorname{Hom}_{R \otimes C}(R \otimes \mathfrak{g}, R \otimes C) \cong R \otimes \operatorname{Hom}_{C}(\mathfrak{g}, C), \operatorname{Hom}_{R \otimes C}(R \otimes \mathfrak{g}, R) \cong R \otimes$ $\operatorname{Hom}_{C}(\mathrm{~g}, A)$. Write $\Omega_{R}^{1}=\operatorname{Hom}_{R \otimes C}(R \otimes g, R \otimes C)$. It is clear that the exterior algebra of $\Omega_{R}^{1}$ over $R \otimes C$ may be identified with $R \otimes \Omega$. It is also clear that with this identification the kernel of the exterior differentiation $\Omega_{R}^{1} \rightarrow \Omega_{R}^{1} \wedge \Omega_{R}^{1}$ becomes identified with $R \otimes \Xi$. In the sequence (1), if we replace the extension $C$ over $A$ by 
the extension $R \otimes C$ over $R$, and taking into account these identifications, we get the sequence

$$
0 \longrightarrow R^{*} \longrightarrow(R \otimes C)^{*} \longrightarrow R \otimes \Xi \underset{\delta_{R}}{\longrightarrow} R \otimes \Theta
$$

where $\Theta$ stands for $\operatorname{Hom}_{C}(\mathrm{~g}, A)$. Replacing $R$ by $R \otimes R, R \otimes R \otimes R, \ldots$, we get the sequence of complexes

(3) $0 \rightarrow \mathscr{C}\left(R / A, G_{m}\right) \rightarrow \mathscr{C}\left(R \otimes C / C, G_{m}\right) \rightarrow \mathscr{C}\left(R / A, G_{a}\right) \otimes \Xi \rightarrow \mathscr{C}\left(R / A, G_{a}\right) \otimes \Theta$

where $\mathscr{C}\left(R / A, G_{m}\right), \mathscr{C}\left(R / A, G_{a}\right)$ denote respectively the multiplicative and the additive Amitsur complexes; cf. [14, pp. 284-286].

From now on we assume that $R$ is a $C$-algebra which is faithfully flat as a $C$ module. By Remark (2) of Lemma 14, (3) is an exact sequence of complexes. Let $\AA$ and $\mathbb{C}$ denote respectively the kernel and cokernel of $\mathscr{C}\left(R / A, G_{a}\right) \otimes \Xi$ $\rightarrow \mathscr{C}\left(R / A, G_{a}\right) \otimes \Theta$. We therefore have two exact sequences of complexes

$$
\begin{aligned}
& 0 \rightarrow \mathscr{C}\left(R / A, G_{m}\right) \rightarrow \mathscr{C}\left(R \otimes C / C, G_{m}\right) \rightarrow \mathfrak{\Re} \rightarrow 0, \\
& 0 \rightarrow \mathfrak{\Omega} \rightarrow \mathscr{C}\left(R / A, G_{a}\right) \otimes \Xi \rightarrow \mathfrak{C} \rightarrow 0 .
\end{aligned}
$$

According to [13, Proposition 3.3], the cohomology of $\mathscr{C}\left(R \otimes C / C, G_{m}\right)$ coincides with that of $\mathscr{C}\left(R / C, G_{m}\right)$. According to [13, Lemma 2.2] all cohomology groups of $\mathscr{C}\left(R / A, G_{a}\right) \otimes \Xi$ of dimensions higher than zero are trivial. So we have the following exact sequences

$$
\begin{aligned}
0 \rightarrow A^{*} \rightarrow C^{*} & \rightarrow H^{0} \mathfrak{\Re} \rightarrow H^{1}\left(R / A, G_{m}\right) \rightarrow H^{1}\left(R / C, G_{m}\right) \\
& \rightarrow H^{1} \mathfrak{\Re} \rightarrow H^{2}\left(R / A, G_{m}\right) \rightarrow \cdots, \\
0 & \rightarrow H^{0} \mathfrak{\Re} \rightarrow \Xi \rightarrow H^{0} \mathfrak{C} \rightarrow H^{1} \mathfrak{\Re} \rightarrow 0, \\
0 & \rightarrow H^{t} \mathfrak{C} \rightarrow H^{i+1} \mathfrak{\Uparrow} \rightarrow 0, \quad i>0 .
\end{aligned}
$$

Now $\mathbb{C}$ is a subcomplex of $\mathscr{C}\left(R / A, G_{a}\right) \otimes \Theta$. The latter is acyclic. So

$$
\begin{aligned}
& H^{0} \mathfrak{C}=\mathfrak{\mho}^{0} \cap \Theta, \\
& H^{i} \mathfrak{C}=\left[\mathfrak{\mho}^{i} \cap B^{i}\left(\mathscr{C}\left(R / A, G_{a}\right) \otimes \Theta\right)\right] / B^{i}(\mathfrak{E})
\end{aligned}
$$

where $\mathfrak{E}^{i}$ denotes the $i$-dimensional cochain group of $\mathfrak{E}, B^{i}(X)$ denotes the $i$ dimensional coboundary group of the complex $X$.

In the above exact sequences, if we replace $R$ by $C$ and denote by $\Delta$ the coboundary operator on $\mathscr{C}\left(C / A, G_{a}\right)$, we get the following isomorphisms:

$$
\lambda: H^{1}\left(C / A, G_{m}\right) \cong L(C / A),
$$

(6) $H^{n+1}\left(C / A, G_{m}\right) \cong\left[\left(\right.\right.$ image $\left.\left.\delta_{C^{n}}\right) \cap(\Delta \otimes 1)\left(C^{n-1} \otimes \Theta\right)\right] /(\Delta \otimes 1)\left(\right.$ image $\left.\delta_{C^{n-1}}\right)$,

The isomorphism $\lambda$ is given by $\sum x_{i} \otimes y_{i} \rightarrow-\sum x_{i} d y_{i}$ The isomorphism $\zeta$ is given by $\sum x_{i} \otimes y_{i} \otimes z_{i} \rightarrow \delta_{C}(\omega)$ where $\omega$ is any element in $C \otimes \Xi$ with $(\Delta \otimes 1)(\omega)=$ 
$\left(\sum x_{i} \otimes y_{i} \otimes z_{i}\right)^{-1}\left(\sum x_{i} \otimes y_{i} \otimes d z_{i}\right)$. The groups $H^{n}\left(C / A, G_{m}\right), n>2$, will be shown to be zero later.

5. Regular extensions and Amitsur cohomology. Given $\theta$ in $\operatorname{Hom}_{C}(g, A)$, we now proceed to show that the $C$-module direct sum $C+g$ form a restricted Lie algebra $\mathfrak{l}_{\theta}$ with respect to the commutation $\left[(x, \partial),\left(x^{\prime}, \partial^{\prime}\right)\right]=\left(\partial x^{\prime}-\partial^{\prime} x,\left[\partial, \partial^{\prime}\right]\right)$ and the $p$-map $(x, \partial)^{p}=\left(x^{p}+\partial^{p-1} x+\theta \partial, \partial^{p}\right)$, such that the exact sequence

$$
\begin{gathered}
L_{\varepsilon}: 0 \longrightarrow C \longrightarrow \mathfrak{l}_{\theta} \longrightarrow \mathfrak{g} \rightarrow 0 \\
x \rightarrow(x, 0) \\
(x, \partial) \rightarrow \partial
\end{gathered}
$$

is a regular extension. That $\mathfrak{l}_{\theta}$ form a Lie algebra can be seen from the fact that the commutation on $\mathfrak{l}_{\theta}$ coincides with the one on $C+g$ regarded as a Lie subalgebra of $\operatorname{End}_{A}(C)$. For any $\alpha$ in $A$, it is clear that $(\alpha x, \alpha \partial)^{p}=\alpha^{p}(x, \partial)^{p}$. Moreover, by a simple inductive argument, we have for any positive integer $n$ and any $\left(x^{\prime}, \partial^{\prime}\right)$ in $\mathfrak{l}_{\theta}$, the following formula:

$$
D_{(x, \partial)}^{n}\left(x^{\prime}, \partial^{\prime}\right)=\left(\partial^{n} x^{\prime}+\sum_{i=1}^{n}(-1)^{i} C_{i}^{n} \partial^{n-i} \partial^{\prime} \partial^{i-1} x, D_{\partial}^{n}\left(\partial^{\prime}\right)\right) .
$$

In the special case $n=p$, we have

$$
\begin{aligned}
D_{(x, \partial)}^{p}\left(x^{\prime}, \partial^{\prime}\right) & =\left(\partial^{p} x^{\prime}-\partial^{\prime} \partial^{p-1} x, D_{\partial}^{p}\left(\partial^{\prime}\right)\right) \\
& =\left[\left(x^{p}+\partial^{p-1} x+\theta(\partial), \partial^{p}\right),\left(x^{\prime}, \partial^{\prime}\right)\right]=D_{(x, \partial)^{p}}\left(x^{\prime}, \partial^{\prime}\right) .
\end{aligned}
$$

Now to avoid confusion of notations, let us temporarily write the $p$-map on $\mathfrak{l}_{\theta}$ as $(x, \partial)^{[p]}$. We would like to show that $\left((x, \partial)+\left(x^{\prime}, \partial^{\prime}\right)\right)^{[p]}-(x, \partial)^{[p]}-\left(x^{\prime}, \partial^{\prime}\right)^{[p]}$ is equal to $\left((x, \partial)+\left(x^{\prime}, \partial^{\prime}\right)\right)^{p}-(x, \partial)^{p}-\left(x^{\prime}, \partial^{\prime}\right)^{p}$ in the universal enveloping algebra of $\mathfrak{I}_{\theta}$. By definition

$$
\begin{aligned}
&\left((x, \partial)+\left(x^{\prime}, \partial^{\prime}\right)\right)^{[p]}-(x, \partial)^{[p]}-\left(x^{\prime}, \partial^{\prime}\right)^{[p]} \\
&=\left(\left(x+x^{\prime}\right)^{p}+\left(\partial+\partial^{\prime}\right)^{p-1}\left(x+x^{\prime}\right)+\theta\left(\partial+\partial^{\prime}\right),\left(\partial+\partial^{\prime}\right)^{p}\right) \\
&-\left(x^{p}+\partial^{p-1} x+(\theta \partial), \partial^{p}\right)-\left(x^{\prime p}+\partial^{\prime p-1} x^{\prime}+\left(\theta \partial^{\prime}\right), \partial^{\prime p}\right) \\
&=\left(\left(\partial+\partial^{\prime}\right)^{p-1}\left(x+x^{\prime}\right)-\partial^{p-1} x-\partial^{\prime p-1} x^{\prime},\left(\partial+\partial^{\prime}\right)^{p}-\partial^{p}-\partial^{\prime p}\right) .
\end{aligned}
$$

Now using the formula of Jacobson we have

$$
\begin{aligned}
\left((x, \partial)+\left(x^{\prime}, \partial^{\prime}\right)\right)^{p}-(x, \partial)^{p}-\left(x^{\prime}, \partial^{\prime}\right)^{p}= & \left(\left(x+x^{\prime}\right)^{p}+\left(\partial+\partial^{\prime}\right)^{p-1}\left(x+x^{\prime}\right),\left(\partial+\partial^{\prime}\right)^{p}\right) \\
& -\left(x^{p}+\partial^{p-1} x, \partial^{p}\right)-\left(x^{\prime p}+\partial^{\prime p-1} x^{\prime}, \partial^{\prime p}\right) .
\end{aligned}
$$

This shows $\mathfrak{I}_{\theta}$ is a restricted Lie algebra and we resume our old notation for the p-map on $\mathfrak{I}_{\theta}$.

It is clear that $L_{\theta}$ satisfies the first three regularity requirements. The fourth regularity condition is equivalent to the condition $(c \partial)^{p-1}(c x)=c^{p}\left(\partial^{p-1} x\right)$ $+\left((c \partial)^{p-1} c\right) x, c, x \in C, \partial \in \mathfrak{g}$. Now every $\partial$ in $\mathfrak{g}$ may be regarded as a derivation of the polynomial ring $C[t]$ by letting $\partial t=x$. By [11, Lemma 1] we have $(c \partial)^{p-1}(c x)$ 
$=(c \partial)^{p}(t)=\left(c^{p} \partial^{p}+\left((c \partial)^{p} c\right) \partial\right) t=c^{p}\left(\partial^{p-1} x\right)+\left((c \partial)^{p-1} c\right) x$ as desired. This shows $L_{\theta}$ is a regular extension for any $\theta$ in $\operatorname{Hom}_{C}(\mathrm{~g}, A)$. It is readily verified that $L_{\theta+\theta^{\prime}}$ is equivalent to $L_{\theta}+L_{\theta^{\prime}}$. So the map $\operatorname{Hom}_{C}(\mathfrak{g}, A) \rightarrow \mathscr{E}(\mathfrak{g}, C), \theta \rightarrow L_{\theta}$, is a group homomorphism. We claim that the kernel is exactly the image of $\delta_{1}: \Xi \rightarrow$ $\operatorname{Hom}_{C}(\mathrm{~g}, A)$.

Suppose that $\theta \in \operatorname{Hom}_{C}(\mathrm{~g}, A)$ such that the corresponding regular extension $L_{\theta}$ is trivial. Then there is a $C$-linear restricted Lie algebra homomorphism $\rho: \mathfrak{g} \rightarrow \mathfrak{l}_{\theta}$ such that $\varphi \rho$ is the identity map on $g$. Write $\rho(\partial)=(\omega(\partial), \partial)$. Then $\omega$ is an element of $\Omega^{1}=\operatorname{Hom}_{C}(\mathfrak{g}, C)$. Since $\rho$ is a Lie algebra homomorphism, we have $\omega\left(\left[\partial, \partial^{\prime}\right]\right)$ $=\partial\left(\omega \partial^{\prime}\right)-\partial^{\prime}(\omega \partial)$ which say $d \omega$ is zero $[7$, p. 201, (34)] and hence $\omega$ is actually in $\Xi$. Since $\rho$ is restricted, we have $\left(\omega(\partial)^{p}+\partial^{p-1}(\omega \partial)+\theta(\partial), \partial^{p}\right)=(\omega(\partial), \partial)^{p}=\left(\omega\left(\partial^{p}\right), \partial^{p}\right)$. So $\theta=\delta_{1}(\omega)$. Conversely if $\theta=\delta_{1} \omega$ for some $\omega$ in $\Xi$, it is easy to verify that the map $\mathfrak{g} \rightarrow \mathfrak{I}_{\theta}, \partial \rightarrow(\omega(\partial), \partial)$ is a $C$-linear restricted Lie algebra homomorphism. So $L_{\theta}$ is trivial.

Since we have a monomorphism $\zeta$ from $H^{2}\left(C / A, G_{m}\right)$ into $\operatorname{Hom}_{C}(\mathrm{~g}, A) /\left(\right.$ image $\left.\delta_{1}\right)$, composing $\zeta$ with $\sigma: \operatorname{Hom}_{C}(\mathfrak{g}, A) /\left(\right.$ image $\left.\delta_{1}\right) \rightarrow \mathscr{E}(\mathfrak{g}, C)$, we therefore have a monomorphism $\mu: H^{2}\left(C / A, G_{m}\right) \rightarrow \mathscr{E}(\mathfrak{g}, C)$.

THEOREM 4. The monomorphism $\mu: H^{2}\left(C / A, G_{m}\right) \rightarrow \mathscr{E}(\mathrm{g}, C)$ is an isomorphism.

THEOREM 5. Every regular extension $0 \rightarrow C \hookrightarrow \stackrel{\varphi}{\rightarrow} \mathfrak{g} \rightarrow 0$ admits a C-linear Lie algebra homomorphism $\rho: \mathfrak{g} \rightarrow \mathfrak{l}$ such that $\varphi \rho$ is the identity map on $\mathfrak{g}$. For any such $\rho$, the map $\theta: \partial \rightarrow \rho(\partial)^{p}-\rho\left(\partial^{p}\right)$ is an element of $\operatorname{Hom}_{C}(g, A)$.

Proof. Let $L$ be a regular extension of $C$ by $g$. Let $R$ be the corresponding central separable $A$-algebra with $C$ as a splitting subalgebra. The proof consists of the following three steps:

(i) A 2-cocycle $u$ will be attached to $R$ and a complete description of $R$ will be given in terms of this $u$.

(ii) Let $-L: 0 \rightarrow C \hookrightarrow \stackrel{\mathfrak{\varphi}}{\rightarrow} \mathfrak{g} \rightarrow 0$ be the regular extension corresponding to $R^{0}$. A $C$-linear Lie algebra homomorphism $\rho: \mathfrak{g} \rightarrow \mathfrak{l}$ such that $\varphi \rho$ is the identity map on $\mathfrak{g}$ will be constructed from the 2-cocycle $u$. This will complete the proof of Theorem 5. (The second statement in Theorem 5 was proved earlier in Theorem 1.)

(iii) Let $\theta: \mathfrak{g} \rightarrow A$ be the map $\partial \rightarrow \rho(\partial)^{p}-\rho\left(\partial^{p}\right)$. The equality $-\theta=\zeta(u)$ will be verified. Since $-L=\sigma(\theta)$, we have $\mu(u)=\sigma \zeta(u)=\sigma(-\theta)=L$ completing the proof of Theorem 4.

(i) Put $M=R / J R$ where $J$ is the kernel of the map $C \otimes C \rightarrow C, x \otimes y \rightarrow x y$. By Lemma 4, there is an additive group isomorphism $\Upsilon: C \otimes M \rightarrow R$ such that $\Lambda\left(c \otimes c^{\prime}\right) \Upsilon=\Upsilon \Lambda\left(c^{\prime} \otimes c\right)$ for all $c$ and $c^{\prime}$ in $C$. Since $M$ is a rank one projective $C$-module, the map $\Lambda: C \rightarrow \operatorname{End}_{C}(M), c \rightarrow \Lambda c$, is an isomorphism because locally it is an isomorphism [5, p. 111, Theorem 1, cf. also p. 177, Exercise 9b]. Let $\pi: C \otimes C \rightarrow C \otimes C$ denote the map $c \otimes c^{\prime} \rightarrow c^{\prime} \otimes c$. We have the following 
sequence of isomorphisms:

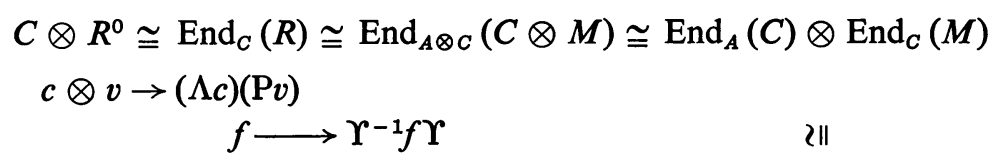

$$
\begin{gathered}
\operatorname{End}_{C \otimes A}(C \otimes C) \cong \operatorname{End}_{A \otimes C}(C \otimes C) \cong \operatorname{End}_{A^{\prime}}(C) \otimes C \\
\pi^{-1} f \pi \longleftarrow \\
\longleftarrow
\end{gathered}
$$

where $\mathrm{P} v$ denote the map $R \rightarrow R$ produced by right multiplication by $v$. Let $\beta: C \otimes R^{0} \rightarrow \operatorname{End}_{C \otimes A}(C \otimes C)$ denote the composite of these isomorphisms. Note that $\beta$ is a $C$-algebra isomorphism and

$$
\beta\left(c \otimes c^{\prime}\right)=\Lambda\left(c \otimes c^{\prime}\right), \quad c, c^{\prime} \in C .
$$

Given any integer $i, 1 \leqq i \leqq n+1$, we shall denote by $\eta_{i}$ the map $\operatorname{End}_{C^{n} \otimes A}\left(C^{n} \otimes C\right)$ $\rightarrow \operatorname{End}_{C^{n+1} \otimes A}\left(C^{n+1} \otimes C\right)$ given by

$$
\left(\eta_{i} f\right)\left(c_{1} \otimes \cdots \otimes c_{n+1} \otimes c\right)=\pi_{i}\left(c_{1} \otimes f\left(c_{i} \otimes \cdots \otimes \hat{c}_{i} \otimes \cdots \otimes c_{n+1} \otimes c\right)\right)
$$

where $\pi_{i}: C^{n+1} \otimes C \rightarrow C^{n+1} \otimes C$ is the map

$$
c_{1} \otimes \cdots \otimes c_{n+1} \otimes c \rightarrow c_{2} \otimes \cdots \otimes c_{i} \otimes c_{1} \otimes c_{i+1} \otimes \cdots \otimes c_{n+1} \otimes c .
$$

If $f=\Lambda z$ for some $z$ in $C^{n+1}$, then $\eta_{i}(\Lambda z)=\Lambda\left(\varepsilon_{i} z\right)$ where $\varepsilon_{i}$ is the $i$ th face operator for the Amitsur complex.

Following [1], [12], we define two $C^{2}$-algebra isomorphisms $\beta_{i}: C^{2} \otimes R^{0}$ $\rightarrow \operatorname{End}_{C^{2} \otimes A}\left(C^{2} \otimes C\right), i=1,2$, by putting

$$
\begin{aligned}
& \beta_{1}\left(c \otimes c^{\prime} \otimes x\right)=(c \otimes 1 \otimes 1) \eta_{1}\left(\beta\left(c^{\prime} \otimes x\right)\right), \\
& \beta_{2}\left(c \otimes c^{\prime} \otimes x\right)=\left(1 \otimes c^{\prime} \otimes 1\right) \eta_{2}(\beta(c \otimes x)) .
\end{aligned}
$$

From (7), we get

$$
\beta_{1}\left(c \otimes c^{\prime} \otimes c^{\prime \prime}\right)=\Lambda\left(c \otimes c^{\prime} \otimes c^{\prime \prime}\right)=\beta_{2}\left(c \otimes c^{\prime} \otimes c^{\prime \prime}\right), \quad c, c^{\prime}, c^{\prime \prime} \in C .
$$

Since $\operatorname{End}_{C^{2} \otimes A}\left(C^{2} \otimes C\right)$ is a central separable $C^{2}$-algebra with $C^{3}$ as a splitting subalgebra, by Lemma $5, \beta_{2} \beta_{1}^{-1}$ is an inner automorphism by $\Lambda z$ with $z$ in $C^{3}$ because the rank one projective class group $P\left(C^{3} / C^{2} \otimes A\right)$ is trivial. From its definition this $z$ satisfies $(\Lambda z)\left(\eta_{1} f\right)(\Lambda z)^{-1}=\eta_{2} f$ for all $f$ in $\beta\left(A \otimes R^{0}\right)$. In other words $\beta\left(A \otimes R^{0}\right)$ form a subring of

$$
R(z)=\left\{f \in \operatorname{End}_{C \otimes A}(C \otimes C) \mid(\Lambda z)\left(\eta_{1} f\right)\left(\Lambda z^{-1}\right)=\eta_{2} f\right\} .
$$

Write $K=\left\{f \in R(z) \mid f \beta(1 \otimes x)=\beta(1 \otimes x) f\right.$ for all $x$ in $\left.R^{0}\right\}$. By [2, Theorem 3.1] the map $R^{0} \otimes K \rightarrow R(z), x \otimes f \rightarrow \beta(1 \otimes x) f$, is an isomorphism. We claim that $K=A$ which implies $R(z)=\beta\left(A \otimes R^{0}\right)$. Given $g$ in $K$, we have $g \beta(c \otimes x)$ $=\beta(c \otimes 1) g \beta(1 \otimes x)=\beta(c \otimes x) g$ for all $c$ in $C$ and $x$ in $R^{0}$. This shows $g$ is in the center of $\operatorname{End}_{C \otimes A}(C \otimes C)$. So $g=\Lambda(c \otimes 1)$ for some $c$ in $C$. Now $\Lambda(1 \otimes c \otimes 1)$ 
$=\eta_{1}(g)=\eta_{2}(g)=\Lambda(c \otimes 1 \otimes 1)$. This is possible only if $c$ is in $A$ because $C$ is faithfully flat as an $A$-module.

Put $u=\left(\varepsilon_{1} z\right)\left(\varepsilon_{2} z\right)^{-1}\left(\varepsilon_{3} z\right)$. It is readily verified that $\Lambda u$ is in the center $C^{3}$ of $\operatorname{End}_{C^{3} \otimes A}\left(C^{3} \otimes C\right)$. If we recall the relation $\varepsilon_{i} \varepsilon_{j}=\varepsilon_{j+1} \varepsilon_{i}$ for $i \leqq j$, we see that $u$ is a 2-cocycle in the multiplicative Amitsur complex. Write $z=\sum a_{i} \otimes b_{i} \otimes c_{i}, a_{i}, b_{i}$, $c_{i} \in C$. Then $u=\left(\sum 1 \otimes a_{i} \otimes b_{i} \otimes c_{i}\right)\left(\sum a_{i} \otimes 1 \otimes b_{i} \otimes c_{i}\right)^{-1}\left(\sum a_{i} \otimes b_{i} \otimes 1 \otimes c_{i}\right)$ as an element in $C^{3} \otimes A$ is equal to (identified with) $\left(\sum 1 \otimes a_{i} \otimes b_{i} c_{i}\right)$ $\cdot\left(\sum a_{i} \otimes 1 \otimes b_{i} c_{i}\right)^{-1}\left(\sum a_{i} \otimes b_{i} \otimes c_{i}\right)$. Put

$$
R(u)=\left\{f \in \operatorname{End}_{C \otimes A}(C \otimes C) \mid(\Lambda u)\left(\eta_{1} f\right)(\Lambda u)^{-1}=\eta_{2} f\right\}
$$

and write $t=\sum a_{i} \otimes b_{i} c_{i}$. For any $f$ in $R(z)$, we have

$$
\begin{aligned}
(\Lambda u) \eta_{1}\left(\left(\Lambda t^{-1}\right) f(\Lambda t)\right)(\Lambda u)^{-1}= & {\left[\left(\Lambda \varepsilon_{1} t\right)\left(\Lambda \varepsilon_{2} t\right)^{-1}(\Lambda z)\right]\left[\left(\Lambda \varepsilon_{1} t\right)^{-1}\left(\eta_{1} f\right)\left(\Lambda \varepsilon_{1} t\right)\right] } \\
& \cdot\left[\left(\Lambda \varepsilon_{1} t\right)^{-1}\left(\Lambda \varepsilon_{2} t\right)(\Lambda z)^{-1}\right] \\
= & \left(\Lambda \varepsilon_{2} t\right)^{-1}\left[(\Lambda z)\left(\eta_{1} f\right)(\Lambda z)^{-1}\right]\left(\Lambda \varepsilon_{2} t\right) \\
= & \eta_{2}\left(\left(\Lambda t^{-1}\right) f(\Lambda t)\right)
\end{aligned}
$$

So $\left(\Lambda t^{-1}\right) f(\Lambda t)$ is an element of $R(u)$ for all $f$ in $R(z)$. Conversely for any $g$ in $R(u)$, $(\Lambda t) g\left(\Lambda t^{-1}\right)$ belongs to $R(z)$. This shows the map $\tau: R(z) \rightarrow R(u), f \rightarrow\left(\Lambda t^{-1}\right) f(\Lambda t)$, is an $A$-algebra isomorphism. It is clear from (7) that $\tau$ leaves the splitting subalgebra $C=\beta(A \otimes C)$ in $R(z)$ pointwise fixed. ( $\tau$ is thus an admissible isomorphism in the terminology of $[8$, p. 38].)

(ii) Now write $u=\sum x_{0} \otimes x_{1} \otimes x_{2}$ and let $\omega$ be an element in $C \otimes \Xi$ whose image under the boundary operator $\Delta \otimes 1$ of $\mathscr{C}\left(C / A, G_{a}\right) \otimes \Xi$ coincides with $\left(\sum x_{0} \otimes x_{1} \otimes x_{2}\right)^{-1}\left(\sum x_{0} \otimes x_{1} \otimes d x_{2}\right)$. For any $\partial$ in $\mathfrak{g}$, we have

$$
\begin{aligned}
(\Lambda u) & \eta_{1}(1 \otimes \partial+\Lambda(\omega \partial))-\eta_{2}(1 \otimes \partial+\Lambda(\omega \partial))(\Lambda u) \\
& =(\Lambda u)\left(1 \otimes 1 \otimes \partial+\Lambda \varepsilon_{1}(\omega \partial)\right)-\left(1 \otimes 1 \otimes \partial+\Lambda \varepsilon_{2}(\omega \partial)\right)(\Lambda u) \\
& =-\Lambda\left(\sum x_{0} \otimes x_{1} \otimes \partial x_{2}\right)+\Lambda u\left(\varepsilon_{1}(\omega \partial)-\varepsilon_{2}(\omega \partial)\right)=0 .
\end{aligned}
$$

This shows $1 \otimes \partial+\Lambda(\omega \partial)$ is an element of $R(u)$ for all $\partial$ in g. If $c$ is in $C$, then $[1 \otimes \partial+\Lambda(\omega \partial), \Lambda(1 \otimes c)]=[1 \otimes \partial, \Lambda(1 \otimes c)]=\Lambda(1 \otimes \partial c)$. So we have a mapping $\rho: \mathfrak{g} \rightarrow \mathfrak{l}, \partial \rightarrow 1 \otimes \partial+\Lambda(\omega \partial)$. It is clear that $\varphi \rho$ is the identity map on $\mathfrak{g}$ and $\rho$ is $C$-linear. Moreover

$$
\begin{aligned}
{[1 \otimes \partial+} & \left.\Lambda(\omega \partial), 1 \otimes \partial^{\prime}+\Lambda\left(\omega \partial^{\prime}\right)\right] \\
& =\left[1 \otimes \partial, 1 \otimes \partial^{\prime}\right]+\left[1 \otimes \partial, \Lambda\left(\omega \partial^{\prime}\right)\right]+\left[\Lambda(\omega \partial), 1 \otimes \partial^{\prime}\right] \\
& =1 \otimes\left[\partial, \partial^{\prime}\right]+\Lambda\left((1 \otimes \partial)\left(\omega \partial^{\prime}\right)-\left(1 \otimes \partial^{\prime}\right)(\omega \partial)\right) \\
& =1 \otimes\left[\partial, \partial^{\prime}\right]+\Lambda \omega\left(\left[\partial, \partial^{\prime}\right]\right) .
\end{aligned}
$$

The last equality holds because $d \omega=0$, cf. [7, p. 201 (34)]. This shows $\rho$ is a Lie algebra homomorphism. The proof for Theorem 5 is therefore complete. 
(iii) Finally, we have

$$
\begin{aligned}
\theta(\partial) & =\rho(\partial)^{p}-\rho\left(\partial^{p}\right)=(1 \otimes \partial+\Lambda(\omega \partial))^{p}-\left(1 \otimes \partial^{p}+\Lambda \omega\left(\partial^{p}\right)\right) \\
& =1 \otimes \partial^{p}+\Lambda\left(\left(1 \otimes \partial^{p-1}\right)(\omega \partial)+(\omega \partial)^{p}\right)-\left(1 \otimes \partial^{p}+\Lambda \omega\left(\partial^{p}\right)\right) \\
& =\Lambda\left(\left(1 \otimes \partial^{p-1}\right)(\omega \partial)+(\omega \partial)^{p}-\omega\left(\partial^{p}\right)\right)=-\Lambda\left(\delta_{C} \omega\right)(\partial)
\end{aligned}
$$

completing the proof of Theorem 4.

COROLlaRy 1. Let $R$ be a central separable A-algebra with $C$ as a splitting subalgebra. Then there is a 2-cocycle $u$ in the multiplicative Amitsur complex for $C$ over $A$ such that $R$ is isomorphic to $R(u) \equiv\left\{f \in \operatorname{End}_{C \otimes A}(C \otimes C) \mid(\Lambda u)\left(\eta_{1} f\right)\left(\Lambda u^{-1}\right)=\eta_{2} f\right\}$ by an A-algebra isomorphism which leaves $C$ pointwise fixed.

Corollary 2. The map $\sigma: \operatorname{Hom}_{C}(\mathfrak{g}, A) /\left(\right.$ image $\left.\delta_{1}\right) \rightarrow \mathscr{E}(\mathfrak{g}, C)$ is an isomorphism.

REMARK. Corollary 2 for the case $C$ is a field is due to Hochschild [11, Theorem 7]. Theorem 4 for the case $C$ is a field is due to Rosenberg and Zelinsky [12, Theorem 6].

TheOREM 6. $H^{i+2}\left(C / A, G_{m}\right) \cong \mathscr{E}\left(C^{i} \otimes \mathrm{g}, C^{i+1}\right) \cong\left[C^{i} \otimes \operatorname{Hom}_{C}(\mathrm{~g}, A)\right] /\left(\right.$ image $\left.\delta_{C^{i}}\right)$ is equal to zero for all $i>0$.

Proof. It follows from Corollary 2 to Theorem 4 that we have the equality

$$
\text { (image } \left.\delta_{C}\right) \cap \operatorname{Hom}_{C}(\mathfrak{g}, A)=\operatorname{Hom}_{C}(\mathfrak{g}, A) \text {. }
$$

Now let $\varepsilon_{j}: C^{i} \rightarrow C^{i+1}$ be the map $x_{1} \otimes \ldots \otimes x_{i} \rightarrow x_{1} \otimes \ldots \otimes x_{j-1} \otimes 1 \otimes x_{j}$ $\otimes \ldots \otimes x_{i}, 1 \leqq j \leqq i+1$. If in the above equality, we replace $C$ over $A$ by $C^{i+1}$ over $\varepsilon_{j} C^{i}$, and $\mathfrak{g}$ by $\varepsilon_{j} C^{i} \otimes \mathfrak{g}$, we get, after identifications $\left(\varepsilon_{j} C^{i}\right) \otimes \operatorname{Hom}_{C}(\mathfrak{g}, A)$ $\subset$ (image $\delta_{C^{i+1}}$ ). In view of the isomorphism (6) given in $\S 4$, we therefore get

$$
H^{i+2}\left(C / A, G_{m}\right) \cong(\Delta \otimes 1)\left(C^{i} \otimes \operatorname{Hom}_{C}(g, A)\right) /(\Delta \otimes 1)\left(\text { image } \delta_{C^{i}}\right) \text {. }
$$

But given any $\theta$ in $C^{i} \otimes \operatorname{Hom}_{C}(\mathfrak{g}, A)$, if $(\Delta \otimes 1) \theta$ is zero, then $\theta$ is of the form $(\Delta \otimes 1)\left(\theta^{\prime}\right)$ for some $\theta^{\prime}$ in $C^{i-1} \otimes \operatorname{Hom}_{C}(g, A)$. In particular $\theta$ must belong to (image $\delta_{C^{i}}$ ). If we consider the kernel-cokernel sequence of the following commutative diagram with exact rows:

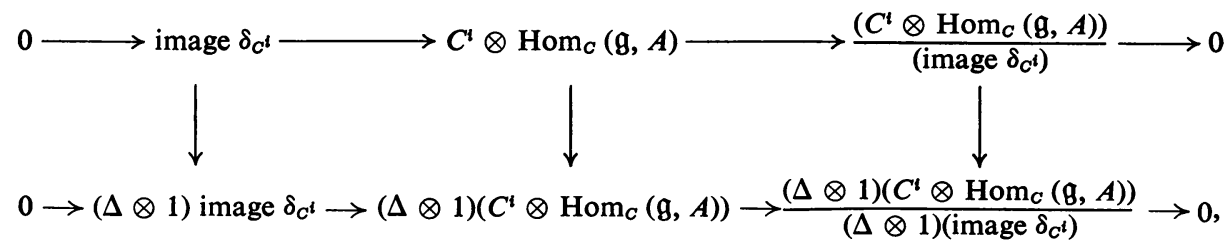

we see that $H^{i+2}\left(C / A, G_{m}\right)$ is isomorphic to $\left(C^{i} \otimes \operatorname{Hom}_{C}(\mathfrak{g}, A)\right) /\left(\right.$ image $\left.\delta_{C^{i}}\right)$ which is isomorphic to $\mathscr{E}\left(C^{i} \otimes \mathfrak{g}, C^{i+1}\right)$. Now the map $C^{n-1} \rightarrow C, x_{1} \otimes \cdots \otimes x_{n-1}$ $\rightarrow x_{1} \cdots x_{n-1}$, is an epimorphism with nilpotent kernel. So when $n>2$

$$
\begin{aligned}
H^{n}\left(C / A, G_{m}\right) & =\mathscr{E}\left(C^{n-2} \otimes \mathfrak{g}, C^{n-1}\right)=H^{2}\left(C^{n-1} / C^{n-2} \otimes A, G_{m}\right) \\
& =H^{2}\left(C / C, G_{m}\right)=0 .
\end{aligned}
$$

The third equality follows from [13, Theorem 3.4]. 
According to [13, Theorem 4.3] if $R$ is a (commutative) $C$-algebra which is finitely generated projective as a $C$-module, then there is an exact sequence

$$
\cdots \rightarrow H^{n-1}\left(R / C, G_{m}\right) \rightarrow H^{n}\left(C / A, G_{m}\right) \rightarrow H^{n}\left(R / A, G_{m}\right) \rightarrow H^{n}\left(R / C, G_{m}\right) \rightarrow \cdots
$$

In view of Theorems 3,4 , and 6 , the following sequence

$$
0 \rightarrow P(C / A) \rightarrow P(R / A) \rightarrow P(R / C) \rightarrow \mathscr{A}(C / A) \rightarrow \mathscr{A}(R / A) \rightarrow \mathscr{A}(R / C) \rightarrow 0
$$

is therefore exact.

COROllary. Let $R$ be a commutative $C$-algebra such that $R$ is finitely generated projective as a $C$-module, then the sequence of Brauer groups

$$
0 \longrightarrow B(C / A) \stackrel{\lambda}{\longrightarrow} B(R / A) \stackrel{\rho}{\longrightarrow} B(R / C) \longrightarrow 0
$$

is exact where $\lambda$ is the lifting homomorphism, $\rho$ is the restriction homomorphism.

Proof. We have a commutative diagram

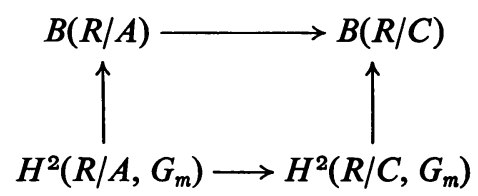

where the horizontal maps are the restriction maps, the vertical maps are defined as in [12, Theorem 2]. By Theorems 3, 4, and [8, Theorem 2.14] the vertical mappings are epimorphisms. By Theorem 6 and [13, Theorem 4.3] the lower horizontal map is also an epimorphism. So the upper horizontal map must be an epimorphism. The rest of the corollary follows from the definition of $B(C / A)$.

REMARK. The above corollary for the case $C$ is a field is due to Hochschild [10, Theorem 5]. Theorem 6 for the case $C$ is a field is due to Berkson [4, Theorem 4]. A proof for Theorem 6 for the case $C$ admits a $p$-basis over $A$ is given in [17, Theorem 3.6].

\section{BIBLIOGRAPHY}

1. S. A. Amitsur, Simple algebras and cohomology groups of arbitrary fields, Trans. Amer. Math. Soc. 90 (1959), 73-112. MR 21 \#78.

2. M. Auslander and $\mathrm{O}$. Goldman, The Brauer group of a commutative ring, Trans. Amer. Math. Soc. 97 (1960), 367-409. MR 22 \#12130.

3. H. Bass, Algebraic K-theory, Math. Lecture Note Series, Benjamin, New York, 1968.

4. A. J. Berkson, On Amitsur's complex and restricted Lie algebras, Trans. Amer. Math. Soc. 109 (1963), 430-443. MR 28 \#2138.

5. N. Bourbaki, Algèbre commutative. Chaps. 1, 2, Actualités Sci. Indust., no. 1290, Hermann, Paris, 1961. MR 36 \#146.

6. H. Cartan and S. Eilenberg, Homological algebra, Princeton Univ. Press, Princeton, N. J., 1956. MR 17, 1040. 
7. P. Cartier, Questions de rationalité des diviseurs en géométrie algébrique, Bull. Soc. Math. France 86 (1958), 177-251. MR 21 \#4957.

8. S. Chase and A. Rosenberg, Amitsur cohomology and the Brauer group, Mem. Amer. Math. Soc. No. 52 (1965), 34-79. MR 33 \#4119.

9. A. Grothendieck and J. Dieudonné, Éléments de géométrie algébrique. I, Inst. Hautes Études Sci. Publ. Math. No. 4 (1960). MR 36 \#177a.

10. G. Hochschild, Restricted Lie algebras and simple associative algebras of characteristic $p$, Trans. Amer. Math. Soc. 80 (1955), 135-147. MR 17, 282.

11. - Simple algebras with purely inseparable splitting fields of exponent one, Trans. Amer. Math. Soc. 79 (1955), 477-489. MR 17, 61.

12. A. Rosenberg and D. Zelinsky, On Amitsur's complex, Trans. Amer. Math. Soc. 97 (1960), 327-356. MR 22 \#12129.

13. — Amitsur's complex for inseparable fields, Osaka Math. J. 14 (1962), 219-240. MR $26 \# 173$.

14. Shuen Yuan, Theory of p-algebras and the Amitsur cohomology groups for inseparable field extensions, J. Algebra 5 (1967), 280-304. MR 37 \#4128.

15. - On logarithmic derivatives, Bull. Soc. Math. France 96 (1968), 41-52. MR 38 \#5764.

16. - Inseparable Galois theory of exponent one, Trans. Amer. Math. Soc. 149 (1970), 163-170.

17. - On the Brauer groups of local fields, Ann. of Math. (2) 82 (1965), 434-444. MR 32 \#2449.

State University of New York at Buffalo,

AMHERST, NEW YoRK 14226 